\title{
Data on Stretching of Fluid Inclusions in Fluorite and Sphalerite
}

$$
\text { by }
$$

\author{
R. J. Bodnar and P. M. Bethke \\ U. S. Geological Survey \\ Reston, VA. 22092
}

Open-File Report 83-790

This report is preliminary and has not been edited or reviewed for conformity with U. S. Geological Survey standards and nomenclature. 


\section{INTRODUCTION}

The data presented in the following tables represent the results of more than 1,300 homogenization measurements on fluid inclusions in samples of fluorite, sphalerite and 4 measurements on barite which have been systematically overheated beyond their original homogenization temperatures. The interpretation of the data together with a discussion of the "stretching" of the inclusions caused by such overheating and implications concerning the validity of temperatures estimated from homogenization measurements are being published in Economic Geology (Bodnar and Bethke, 1984). The primary data presented herein have been plotted in figures and summarized in tables in that paper, but because they are voluminous they were not published therein. They are presented in this open-file report so that they may be available for examination by those wishing to go back to the original data.

\section{SAMPLE INFORMATION}

Table 1 lists sample information for each individual inclusion measured as part of this study. Each inclusion is identified by a sample number (e.g. PMB B022765) followed by an identifier (e.g. Al) which is unique to each individual inclusion. This information is listed in the first column in Table 1. In the second column the host mineral (either fluorite, sphalerite or barite) is listed. The type of inclusion, either primary or secondary (including pseudosecondary) is listed in column 3 , and a qualitative description of the shape of the inclusion, where noted, is indicated in column 4 . Information on the size of the inclusion, where measured, is listed in column 5 . In the case of fluorite the volume of the inclusion was estimated from the diameter of the vapor bubble, the measured salinity and the original homogenization temperature by the method of Bodnar (Bodnar, 1983). Such measurements are identified in the table by the letter " $V$ " following the value listed. In sphalerite samples most of the inclusions were tabular in shape and the included bubble was flattened. Therefore, for sphalerite, only the bubble diameter is reported in Table 1 , no attempt having been made to estimate the actual volume of the inclusion. Such measurements are identified in the table by the letter "D" following the listed value. The values reported in column 5 of Table 1 have not been rounded to the appropriate significant figure. Because of the many factors involved it is difficult to assign an uncertainty to the volume estimates, but certainly they are no better than the third significant figure. No estimates of volume were made for the four inclusions in barite that were measured. Columns 6 and 7 i ist the values of salinity (expressed in weight percent $\mathrm{NaCl}$ equivalent) and homogenization temperature measured on the inclusion prior to overheating.

Most of the measurements on fluorite were made on material from the $\mathrm{Hill}$ Mine in the southern Illinois fluorspar district (sample PBB 13-59). The remaining measurements on fluorite were made on material from the $O H$ vein in the Creede District, Colorado (samples PMB BO 22765 and PMB JF). All measurements on inclusions in sphalerite (samples PMB BS 23365, NJPX, and TMI) were made on material from the $O H$ vein in the Creede District. All measurements on barite were made from a single sample (OH49 211-212) from the Bulldog Mountain Mine in the Creede District. 


\section{RESULTS OF HEATING TESTS}

The results of the heating tests are listed in Table 2 . The individual inclusions are identified in the first column using the same convention as in Table 1. The nature of the experiment was to first measure the homogenization temperature of each fluid inclusion. This original, or "true", homogenization temperature is listed in the last column of Table 1. Following determination of the "true" homogenization temperature, each inclusion was overheated (heated beyond the "true" homogenization temperature) by a small amount, usually 5 to 10 degrees Celsius. The sample was then returned to room temperature and reheated to measure the homogenization temperature. The sample was then again cooled to room temperature and then overheated an additional 5 to 10 degrees Celsius above the previous maximum temperature obtained. This cyclic process was repeated until either the upper temperature limit of the stage (at that time 300 degrees Celsius) was reached or until the inclusions decrepitated (only a few cases, indicated in Table 2 by the notation "dec"). In a few cases, the inclusion was overheated by a substantial amount (30 to 100 degrees Celsius), cooled to room temperature and then reheated to measure the homogenization temperature.

The results of these tests are listed in Table 2 in the following manner. Following each fluid inclusion number listed in the lefthand column are 8 sets of entries of two columns each. These two columns are labelled at the top of the Table: Toh (for the amount of overheating above the initial homogenization temperature) and dTh (for the difference between the initial homogenization temperature and that measured after the indicated increment of overheating, i.e., Th-observed minus Th-initial). Each set of Toh plus dTh represents one increment of overheating. When more than eight increments of overheating were performed on an inclusion the table is "folded back" on itself and the data for the 9 th increment are listed in the lst data column directly beneath the data for the first increment; the data for the remaining increments following in the 2 nd, $3 r d, \ldots$...

For sample PBB 13-59 W10, the following the value of 100 for dTh indicates that the value fell outside the limits of figure 3 in the Economic Geology paper (Bodnar and Bethke, 1984) and therefore was not plotted on that figure. 


\section{REFERENCES CITED}

Bodnar, R. J., 1983, A method of calculating fluid inclusion volumes based on vapor bubble diameters and $P-V-T-X$ properties of inclusion fluids: Econ. Geol., v. 78, no. 3, p. 535-542.

Bodnar, R. J., and Bethke, P. M., 1984, Systematics of stretching of fluid inclusions I: Fluorite and sphalerite at 1 atmosphere confining pressure: Econ. Geol., v. 79, no. 1, p. 141-161. 


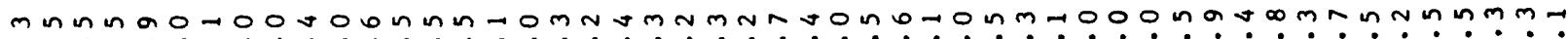

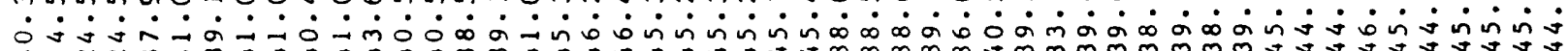
$\checkmark N$ Nm

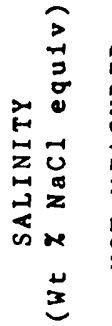

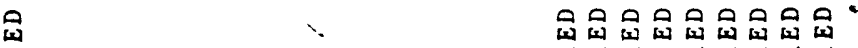

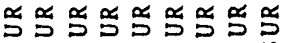

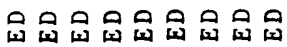

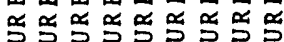
苟
䒴

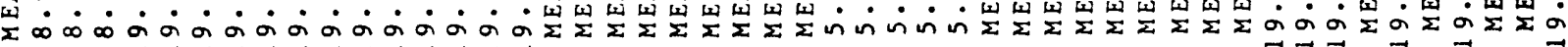 $\stackrel{5}{\circ}$

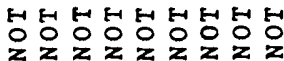

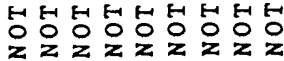

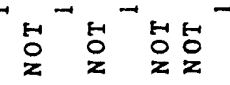

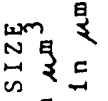

$z \stackrel{5}{=}$

量现

$n>\sum$

30

눙요

$z>\infty$

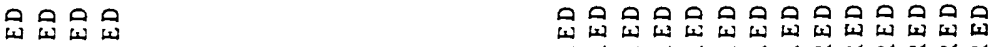

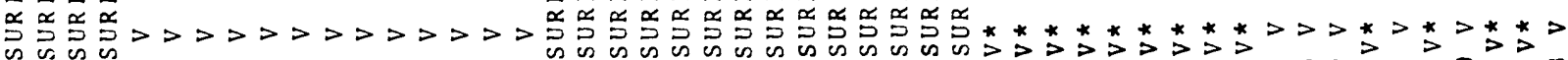

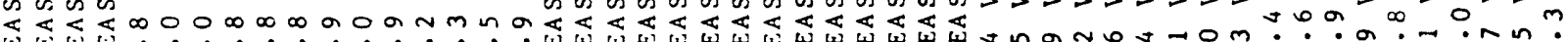

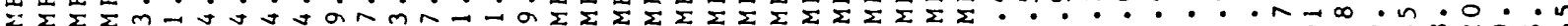

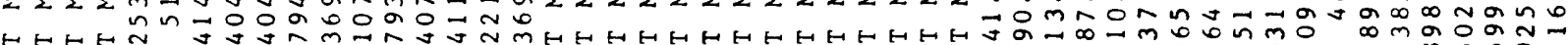

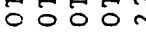
$z \mathrm{z} z \mathrm{z}$

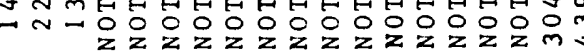

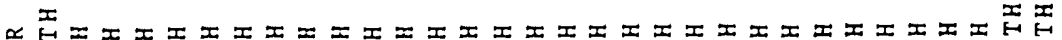

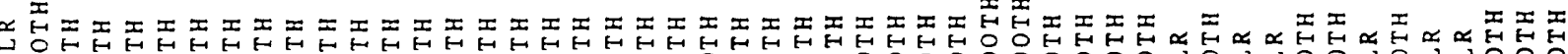

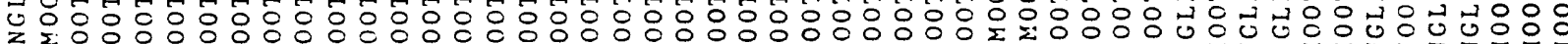

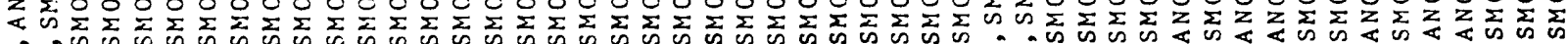

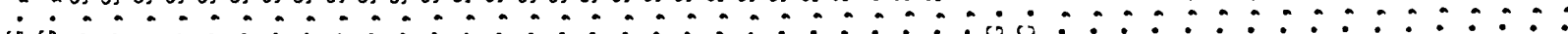

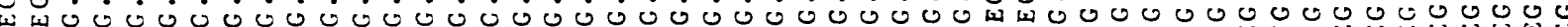
造造

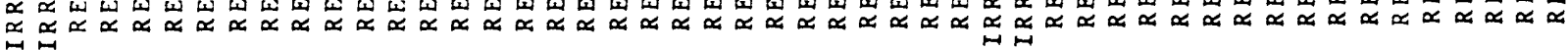

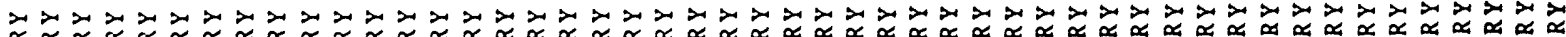

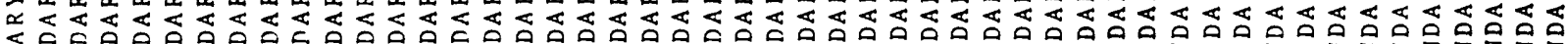

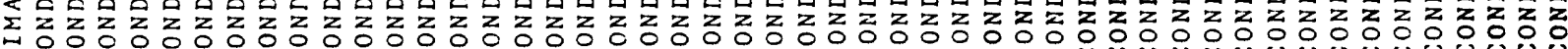

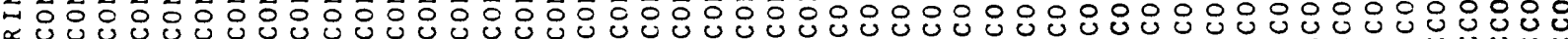

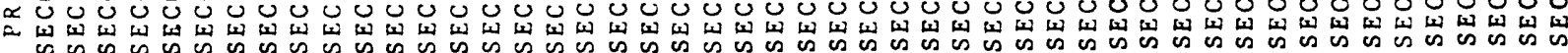

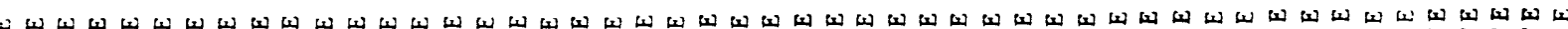

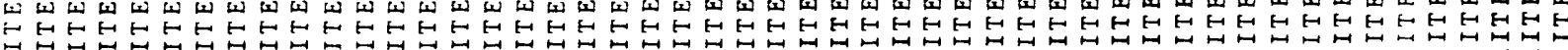

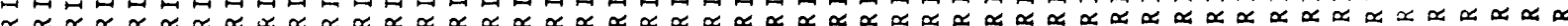

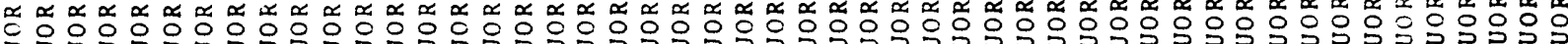

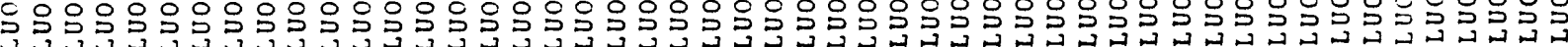

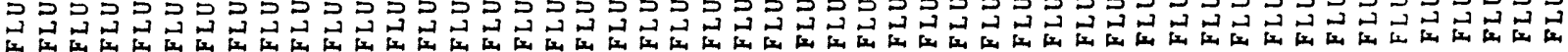

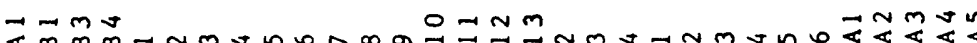

운

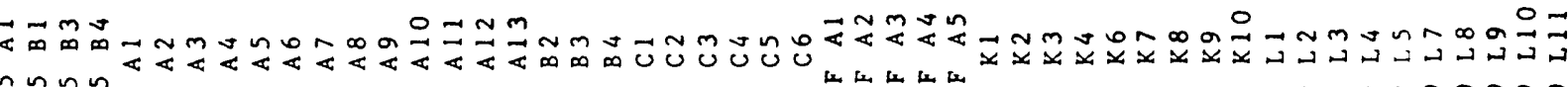

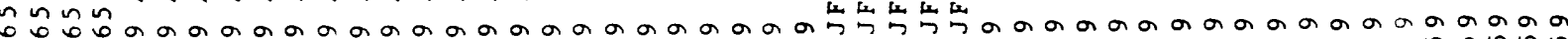

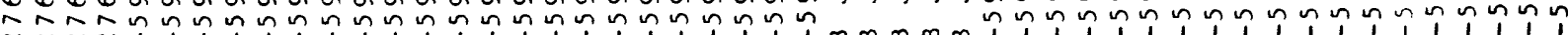

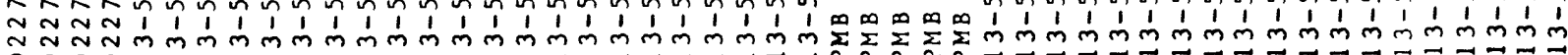
$\circ \circ \circ 0$

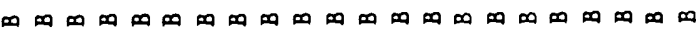

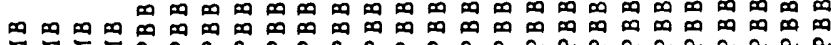

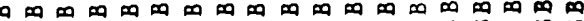

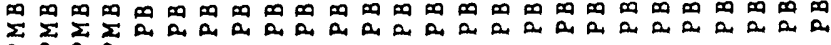
a o a 
mman o mo oa

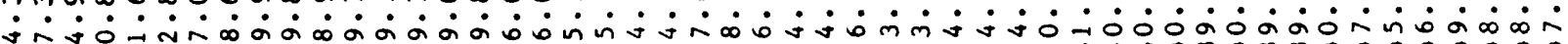
m̄ं

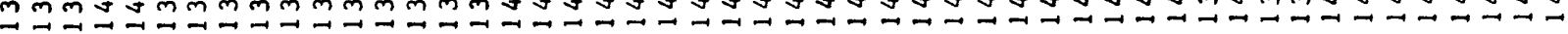

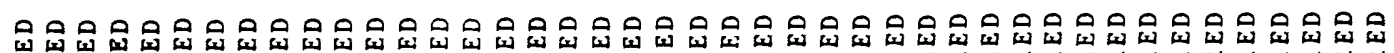

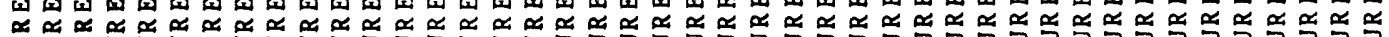

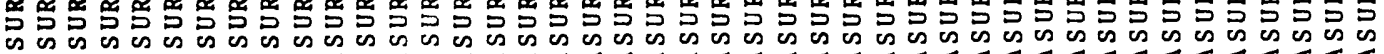

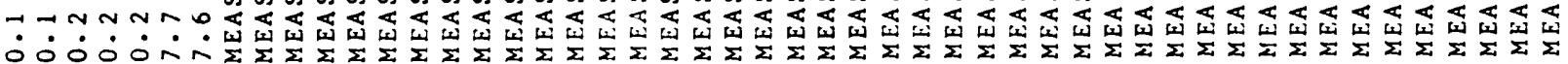
$\operatorname{nn} n \operatorname{nar}$

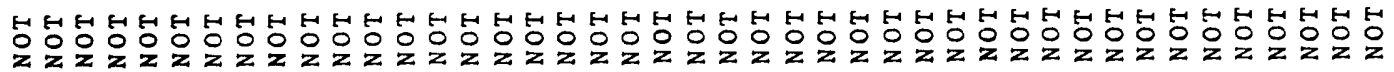

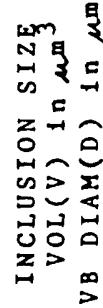

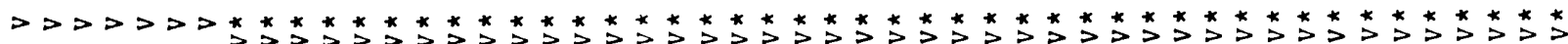
waoo-0 in

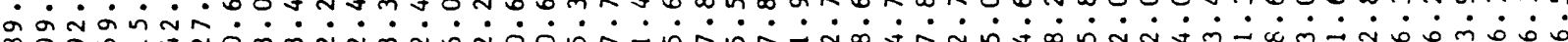

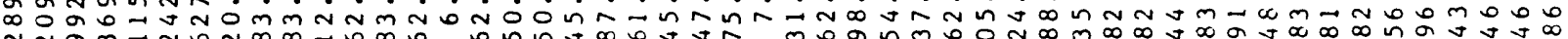

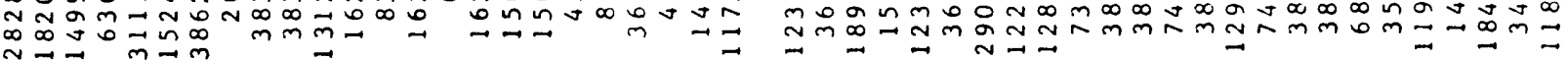

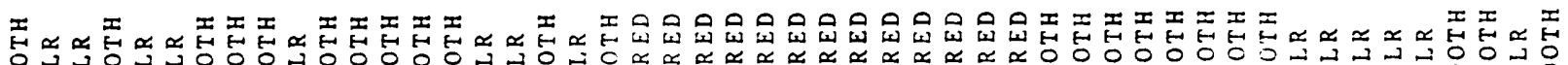

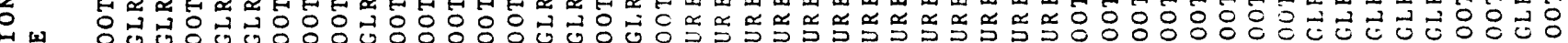
ज的

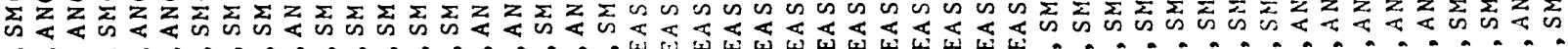

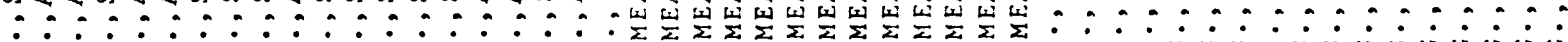

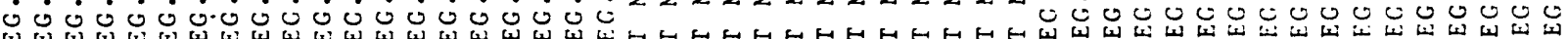

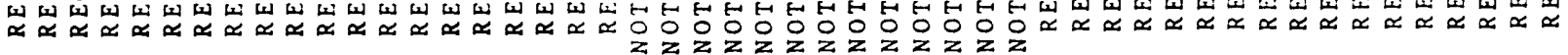

Z

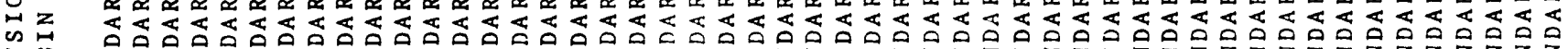

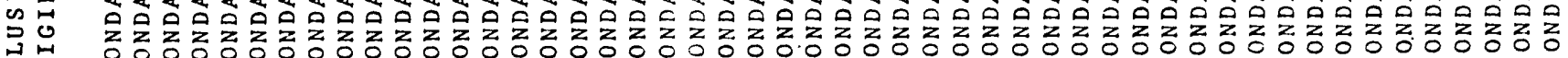
U.

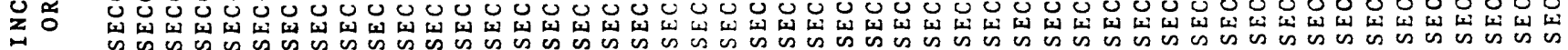

बه

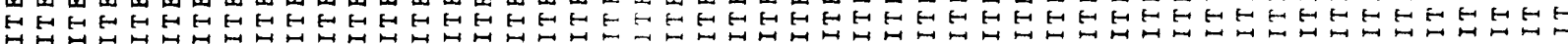

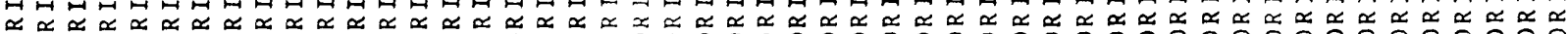

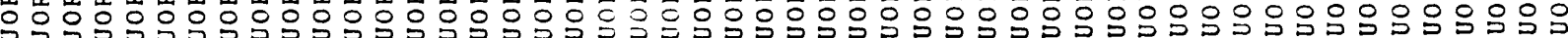

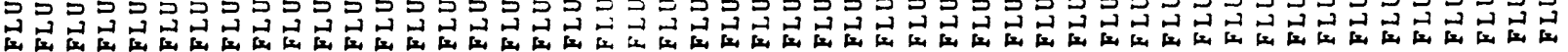

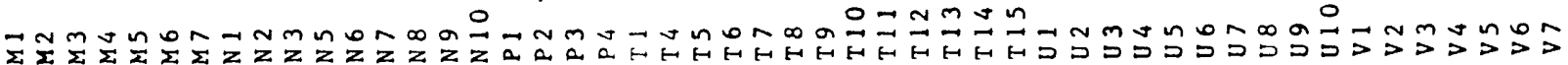

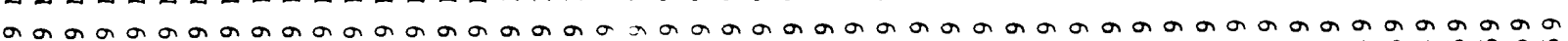

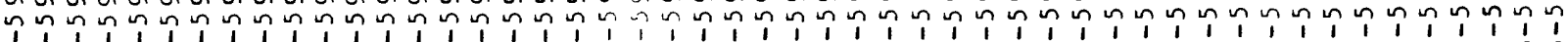

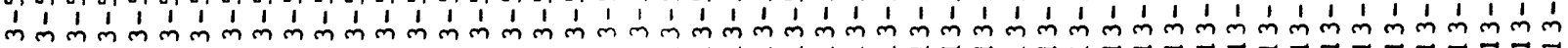

מ

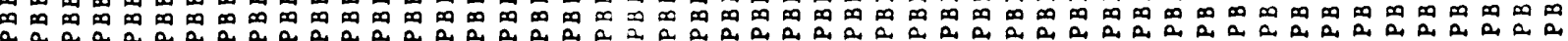




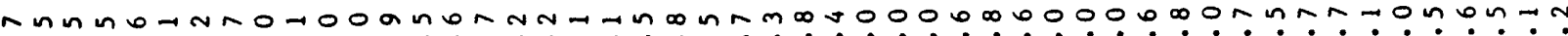

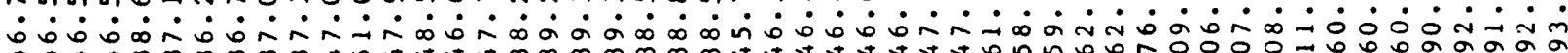

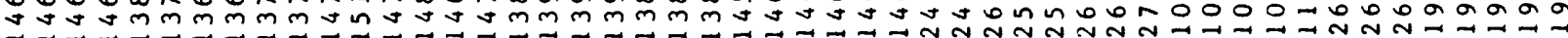

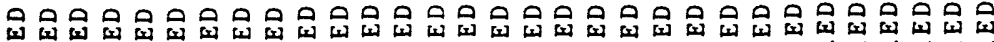

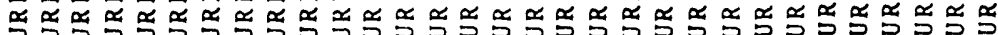

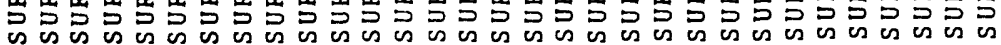

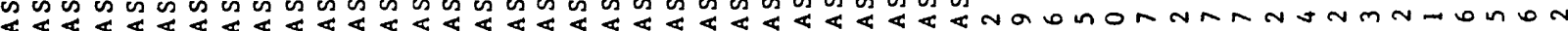

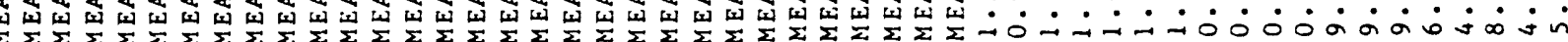

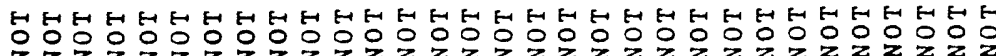

$\underset{3}{3}$

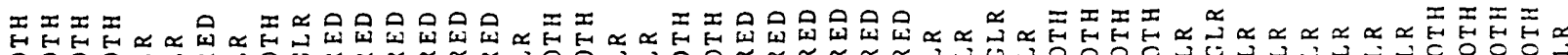

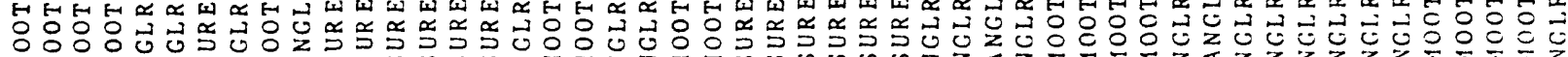

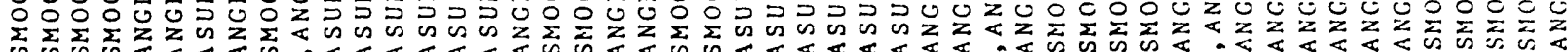

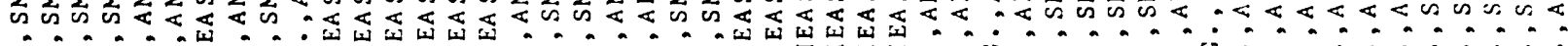

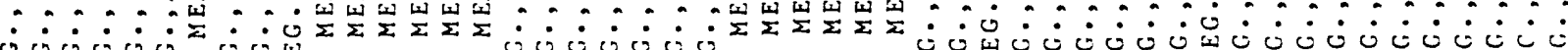

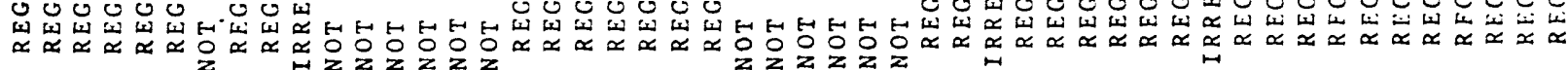

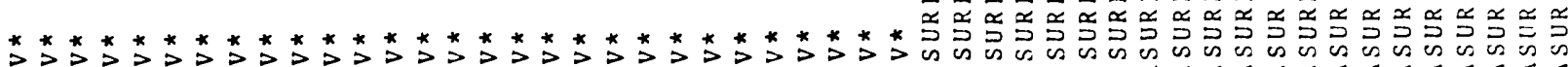

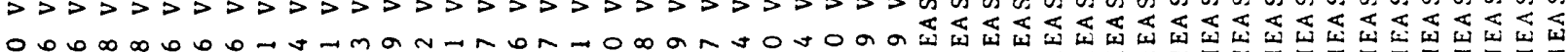
•. nă

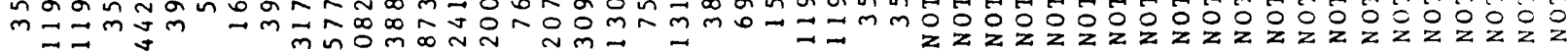
$\sim m=m m$

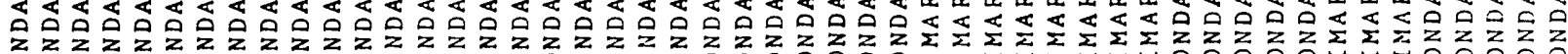

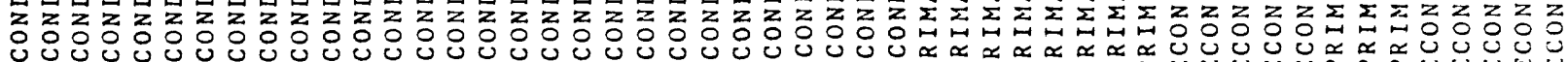
U⿺辶寸

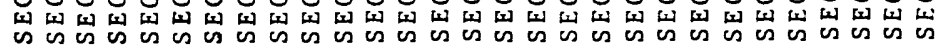

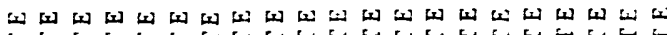
का

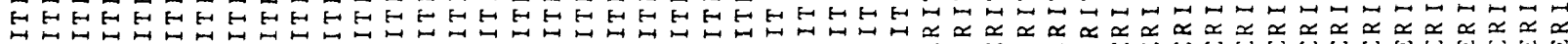

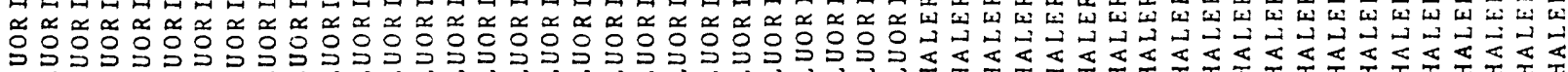

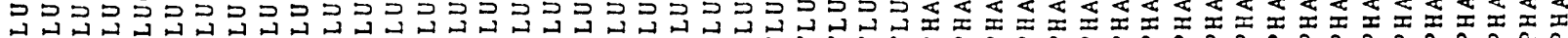

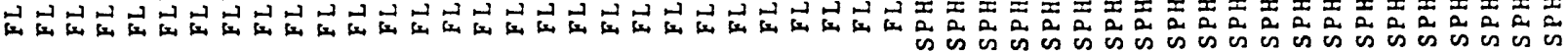

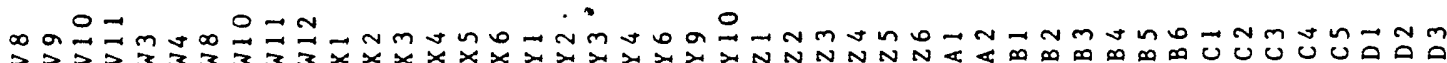

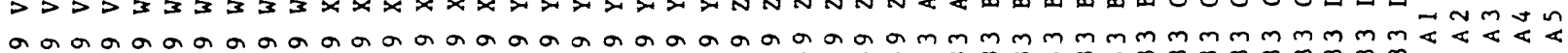

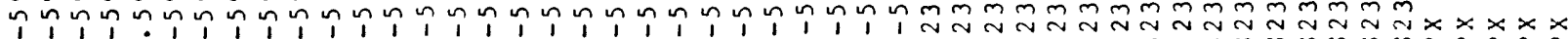

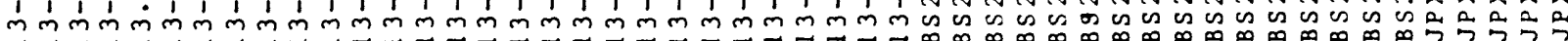
舟

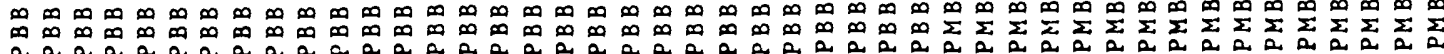




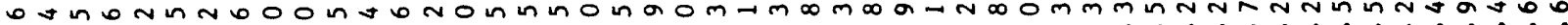

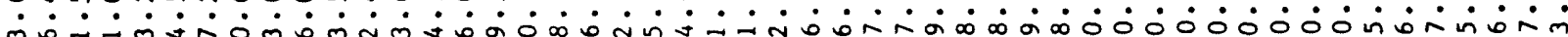

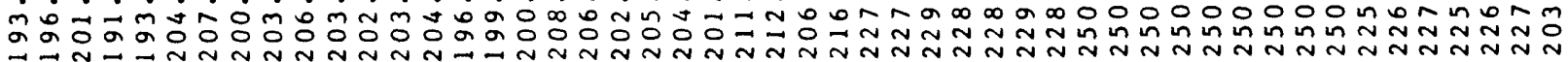

nงtm-un un un

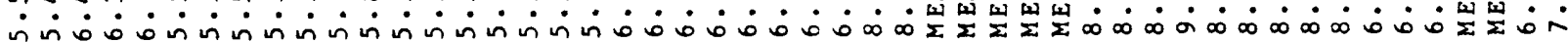

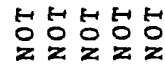

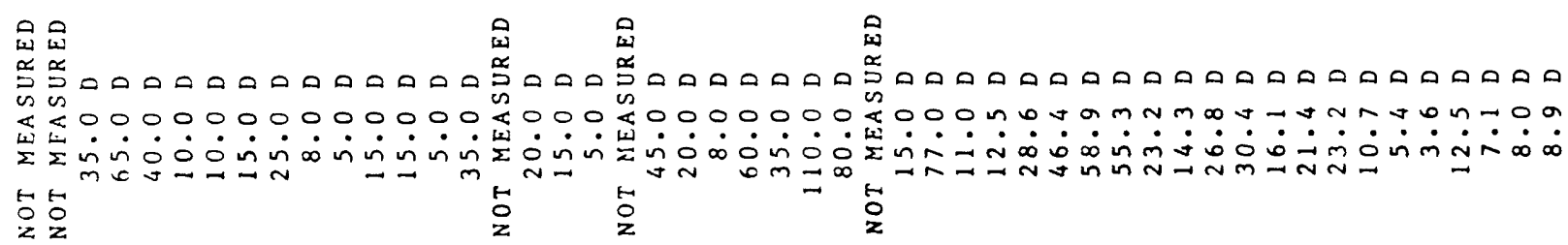

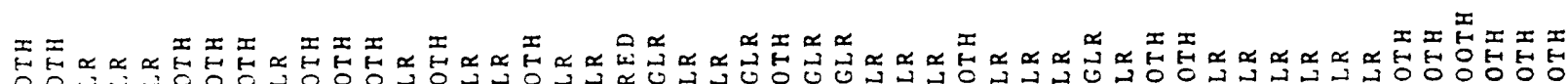

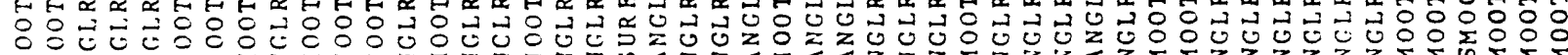

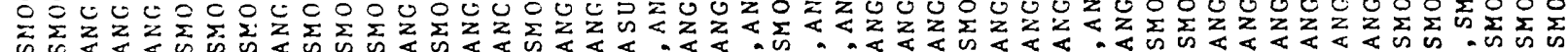

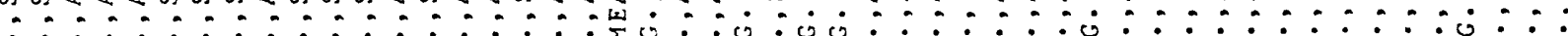

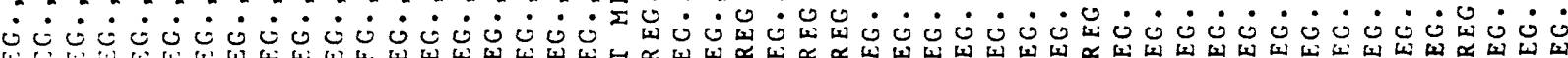

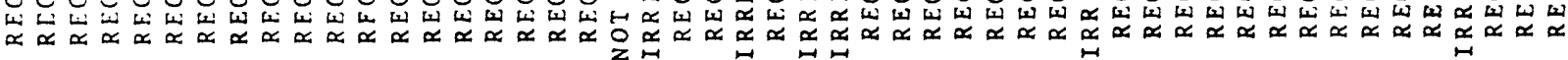

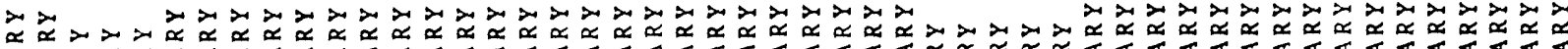

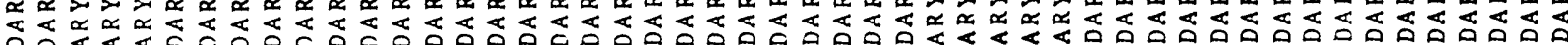

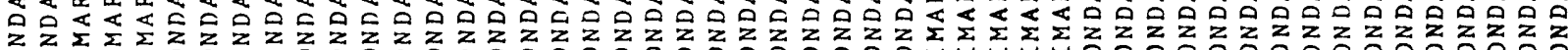

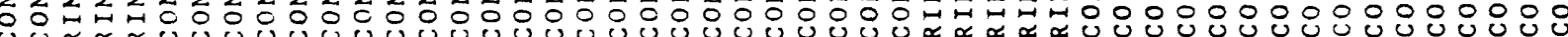

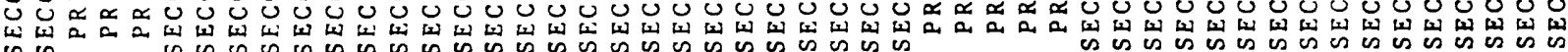

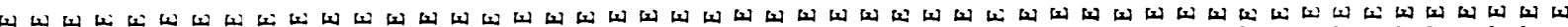

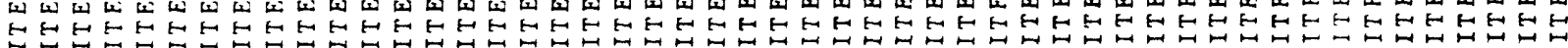

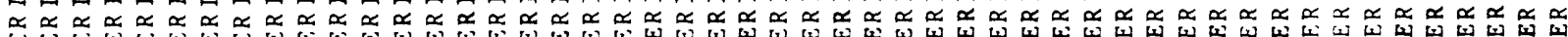

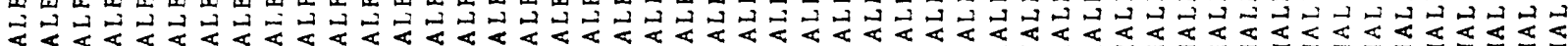

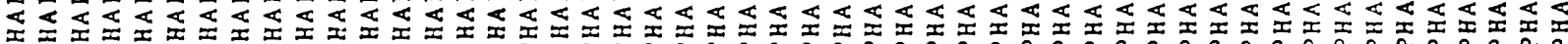

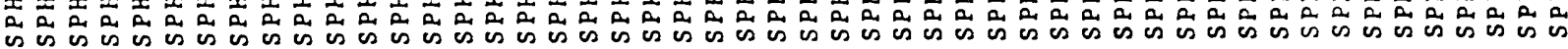

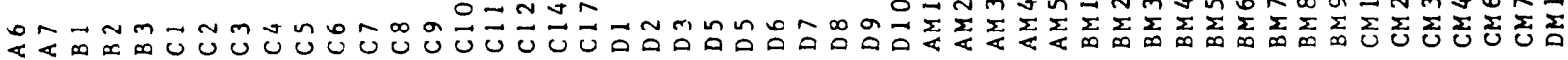
$x \times x \times x \times x \times x \times x \times x \times x \times x \times x \times x \times x \times x \times x \times x \times x \times x \times x \times x \times x \times x \times x \times x \times x \times x \times$

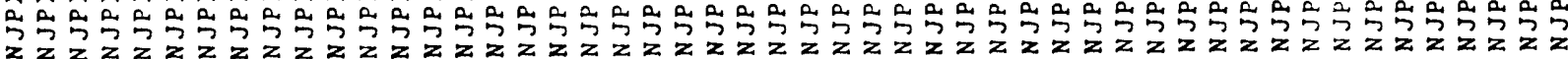




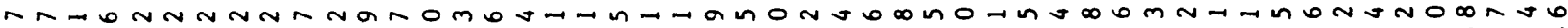
$\dot{0} \dot{0} \dot{0} \dot{0} \dot{0} \dot{0} \dot{0} \dot{0} \dot{0} \dot{0} \dot{0} \dot{0} \dot{0} \dot{0} \dot{0} \dot{0} \dot{0} \dot{0} \dot{0} \dot{0} \dot{0} \dot{0} \dot{0} \dot{0} \dot{0} \dot{0} \dot{0} \dot{0}$

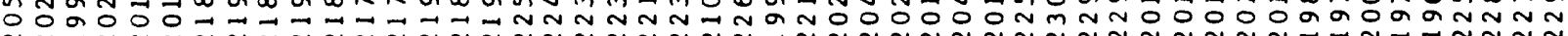

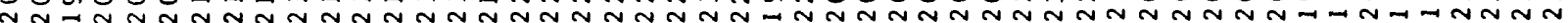

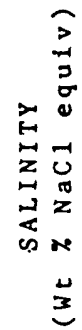

$\stackrel{\ln }{N} \sum_{5}$

is $₹ \Xi$

$z \stackrel{\text { I }}{z-1}$

20

us $>$

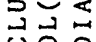

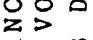

$P^{\infty}$

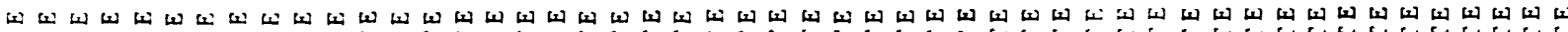

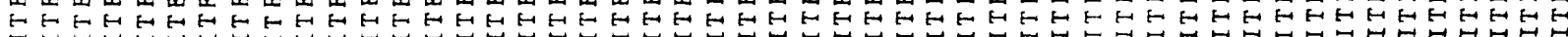

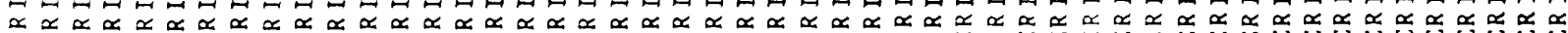

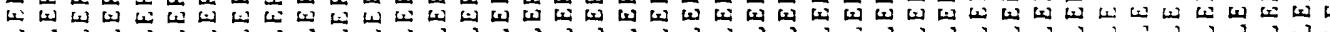

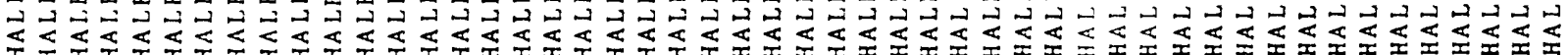
I

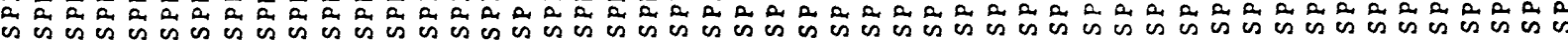

z

$\rightarrow$ 番

$\supset$

U.

$z \underset{z}{3}$

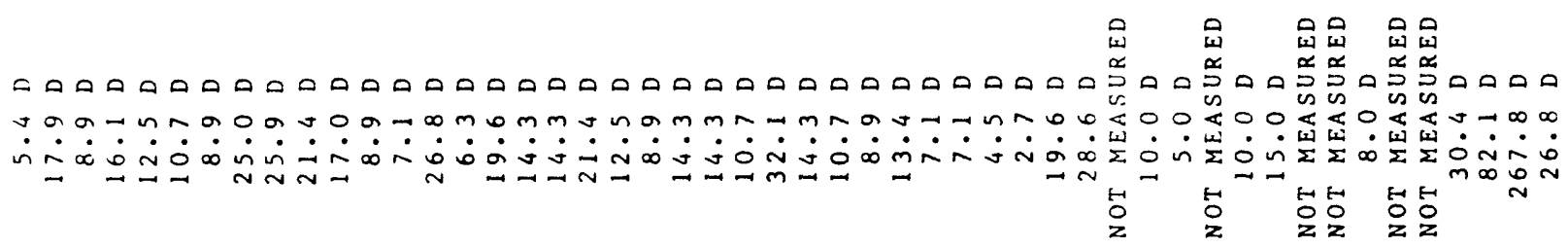

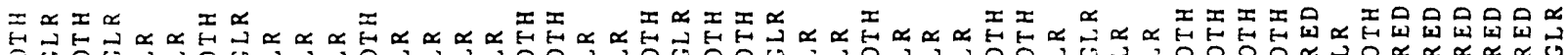

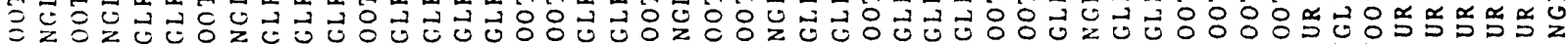

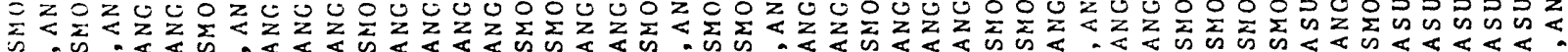

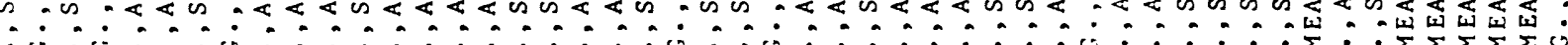
证造

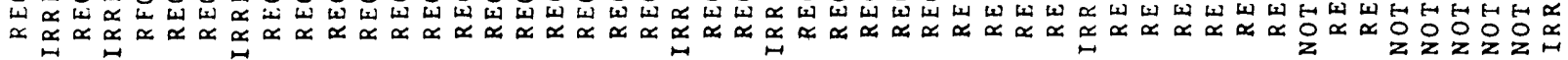

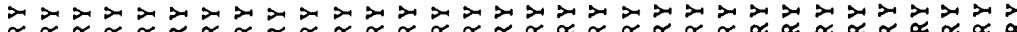

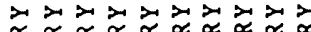

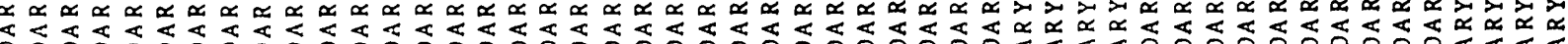

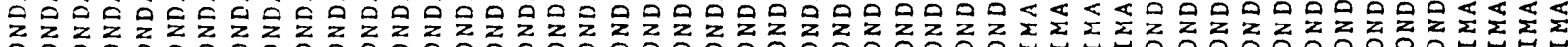

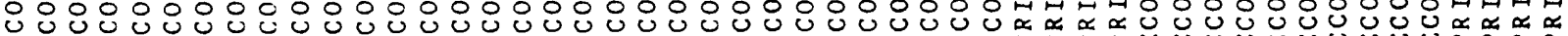

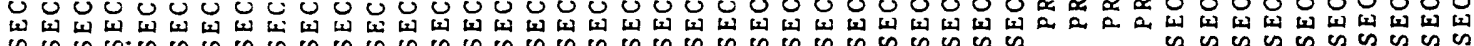

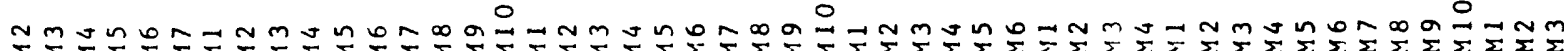

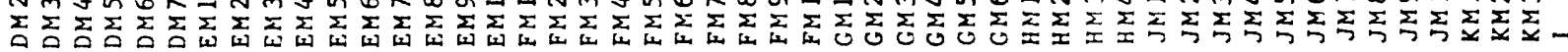

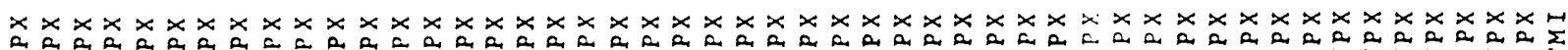

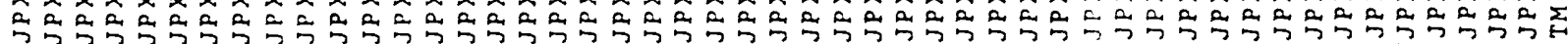

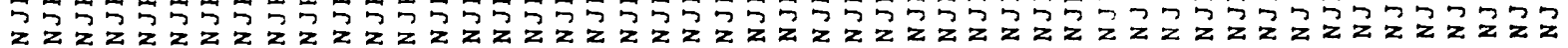




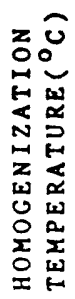

은 $n$ O

$\dot{m} \dot{\sim} \dot{v} \dot{\sim} \dot{\sim}$

Nささ

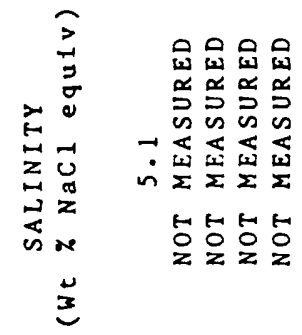

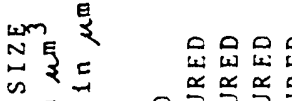

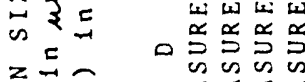

z元

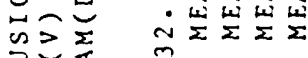

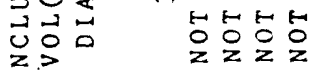

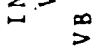

¿
0
0

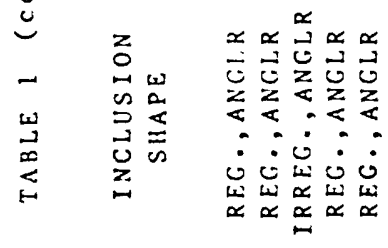

$\dot{0}$
$\vdots$
0
0
0
0
0

-

岕

$\circ$

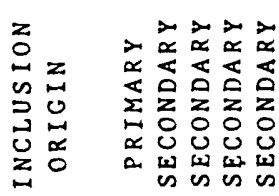

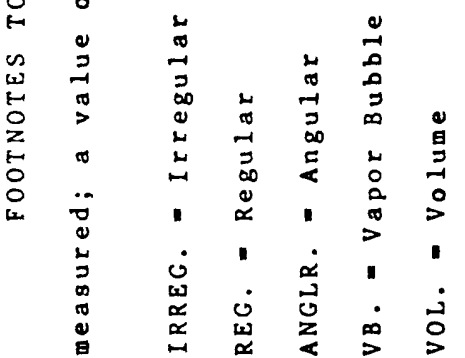

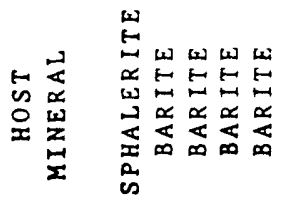

$\overrightarrow{<} \underset{<}{2}<$

$N \sim N N$

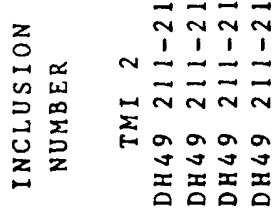




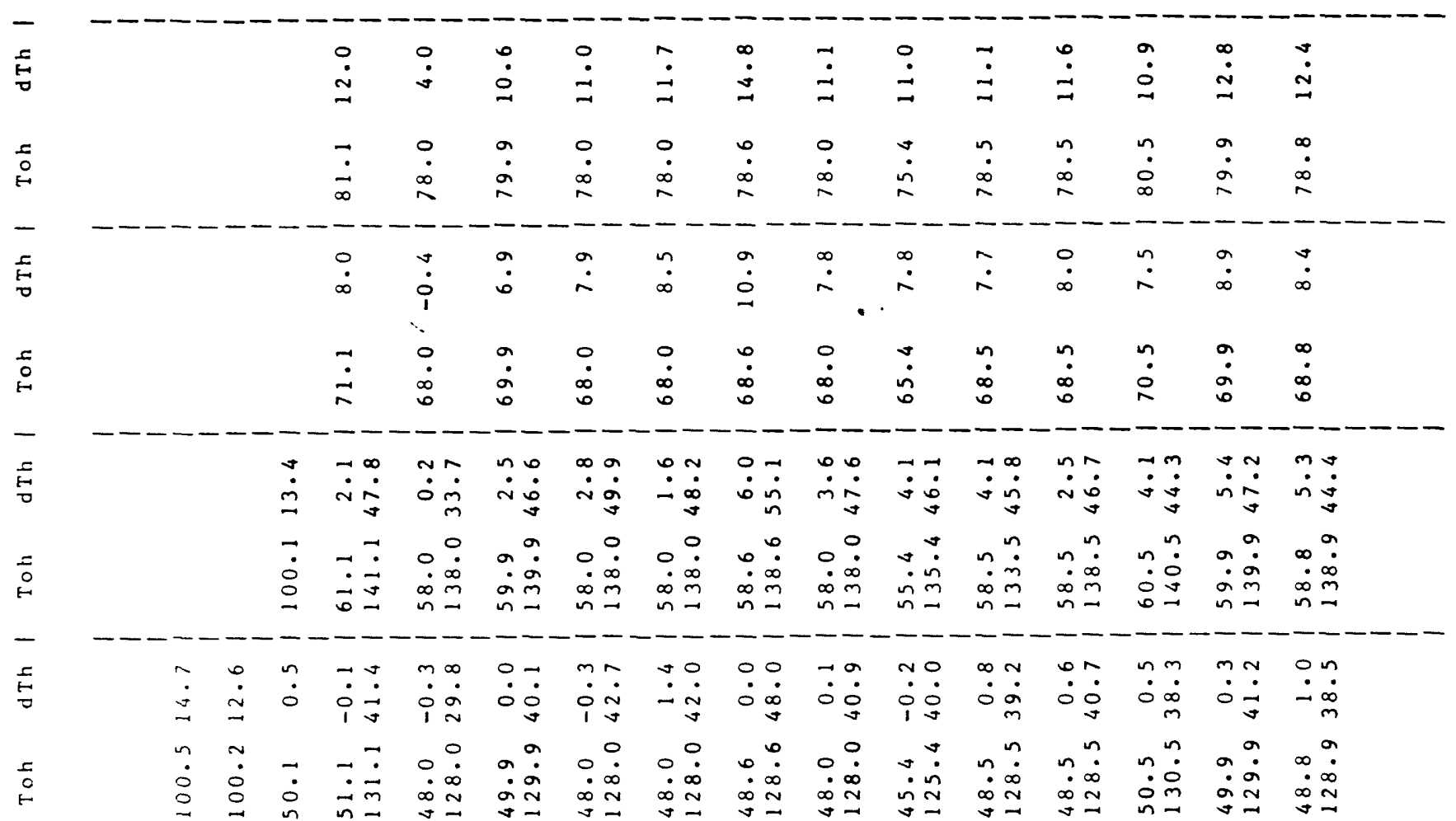

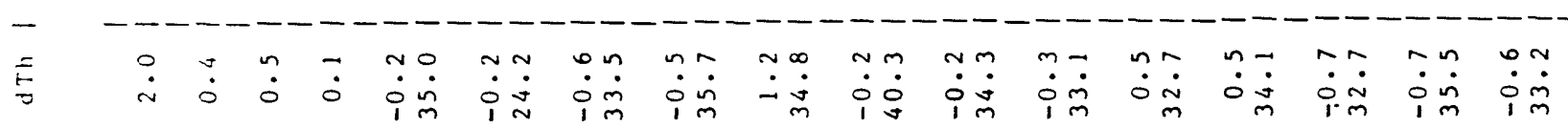

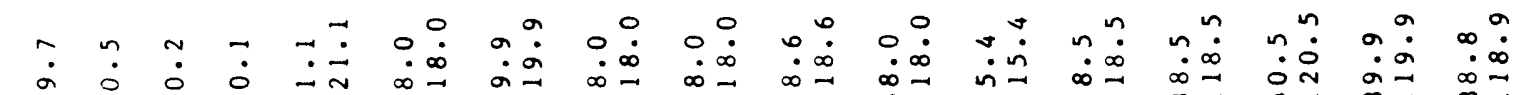

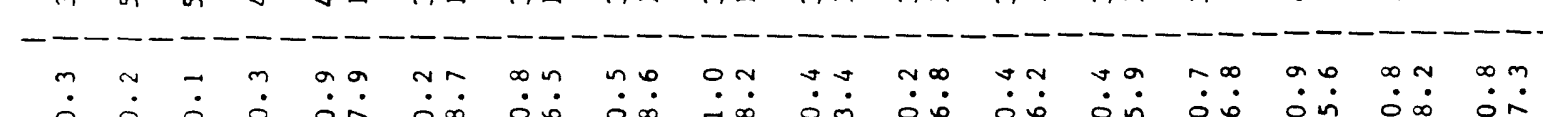

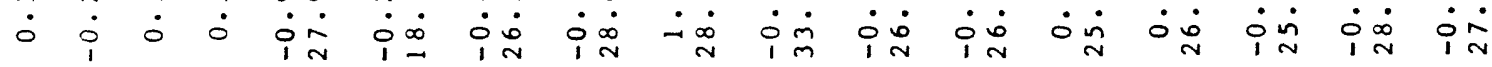

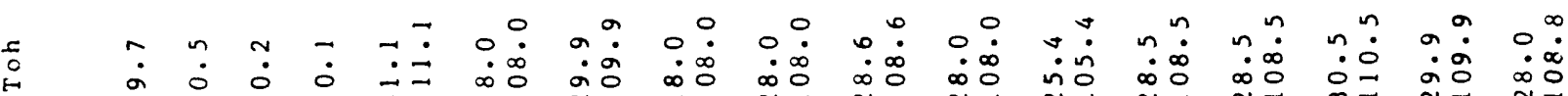

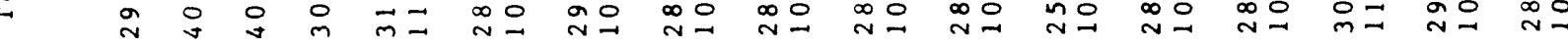

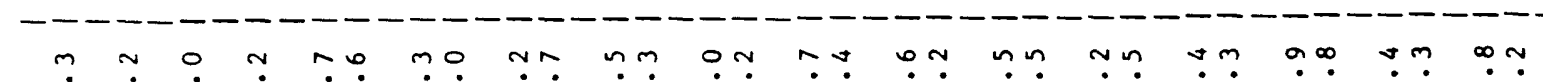

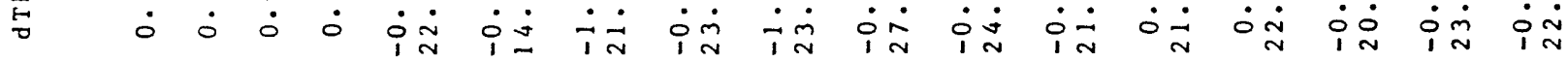

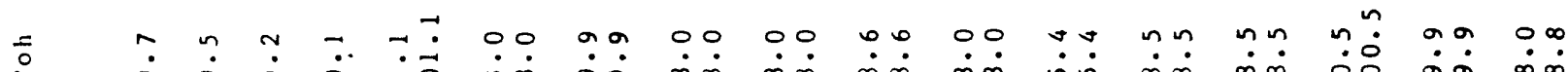

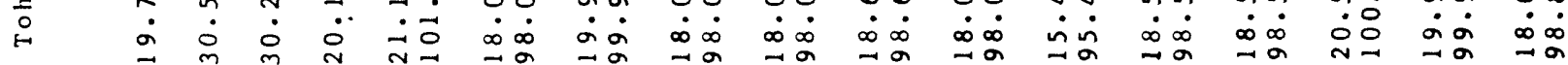
F

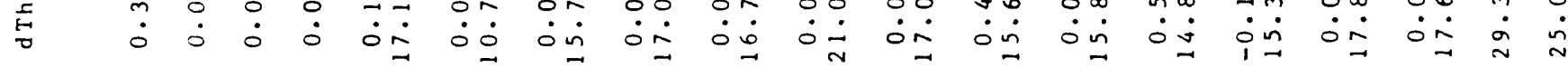

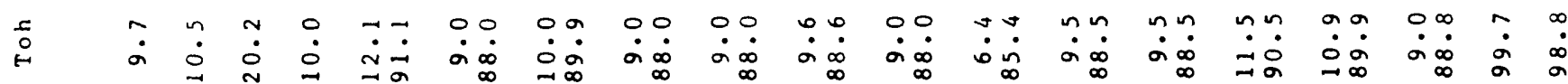

\begin{tabular}{|c|c|c|c|c|c|c|c|c|c|c|c|c|c|c|c|c|c|}
\hline $\overrightarrow{<}$ & $\vec{\infty}$ & $\infty$ & $\vec{\infty}$ & $\overrightarrow{<} \bar{\alpha}$ & $\underset{<}{*}$ & $m_{4}^{m}$ & $\stackrel{<}{<}$ & $\because \ll$ & $\underset{<}{\infty}$ & $\approx<$ & $\mathbb{\infty}_{<}^{\infty}$ & $\frac{a}{<} \frac{\pi}{4}$ & $\frac{00}{<<}$ & $\exists \Xi$ & $\underset{\leftarrow}{\simeq}$ & $\underset{x}{m}$ & $\underset{\infty}{\infty}$ \\
\hline$\approx$ & $\begin{array}{l}n \\
0 \\
N \\
N \\
0\end{array}$ & $\begin{array}{l}n \\
0 \\
N \\
N \\
0\end{array}$ & $\begin{array}{l}\tilde{0} \\
\tilde{N} \\
\tilde{N}\end{array}$ & $\begin{array}{l}\text { on } \\
\text { on } \\
1 \\
m \\
n\end{array}$ & $\begin{array}{l}0 \\
01 \\
11 \\
1 \\
m\end{array}$ & $\begin{array}{ll}0 & \\
0 & n \\
1 & 1 \\
1 & 1\end{array}$ & 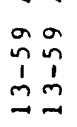 & $\begin{array}{ll}0 & a \\
n & n \\
1 & 1 \\
2 & m\end{array}$ & $\begin{array}{l}0 \\
0 \\
n \\
1 \\
n \\
m \\
n\end{array}$ & 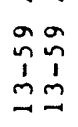 & 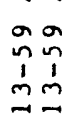 & $\begin{array}{ll}0 & a \\
n & n \\
1 & 1 \\
m & m\end{array}$ & 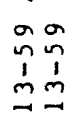 & $\begin{array}{l}09 \\
n \tilde{n} \\
11 \\
m=\end{array}$ & 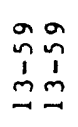 & 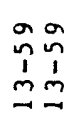 & $\begin{array}{l}a \\
n \\
1 \\
n\end{array}$ \\
\hline$\infty$ & $\sum_{a}^{\infty}$ & $\sum_{n}^{\infty}$ & $\sum_{\infty}^{\infty}$ & $\begin{array}{l}\infty \\
0 \\
0 \\
0 \\
0\end{array}$ & $\begin{array}{l}\infty \\
\infty \\
0 \\
0 \\
0\end{array}$ & $\begin{array}{l}\infty \\
0 \\
\infty \\
\infty \\
0 \\
\infty\end{array}$ & $\begin{array}{l}\infty \\
\text { 吕品 } \\
0\end{array}$ & $\begin{array}{l}\infty \\
\infty \\
\infty \\
\infty\end{array}$ & $\begin{array}{l}\infty \\
0 \\
0 \infty \\
0\end{array}$ & $\begin{array}{l}\infty \\
\infty \\
\infty \\
0 \\
0\end{array}$ & 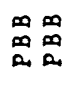 & $\begin{array}{l}\infty \\
\infty \\
\infty \\
0 \\
0\end{array}$ & $\begin{array}{l}\infty \\
\infty \\
\infty \\
0 \\
0\end{array}$ & $\begin{array}{l}\infty \\
\infty \\
\infty \\
\infty \\
a \\
a\end{array}$ & $\begin{array}{l}\text { 品品 } \\
\text { 怘 } \\
0\end{array}$ & $\begin{array}{l}\text { 品品 } \\
\text { a }\end{array}$ & $\begin{array}{l}\infty \\
\infty \\
\infty\end{array}$ \\
\hline
\end{tabular}




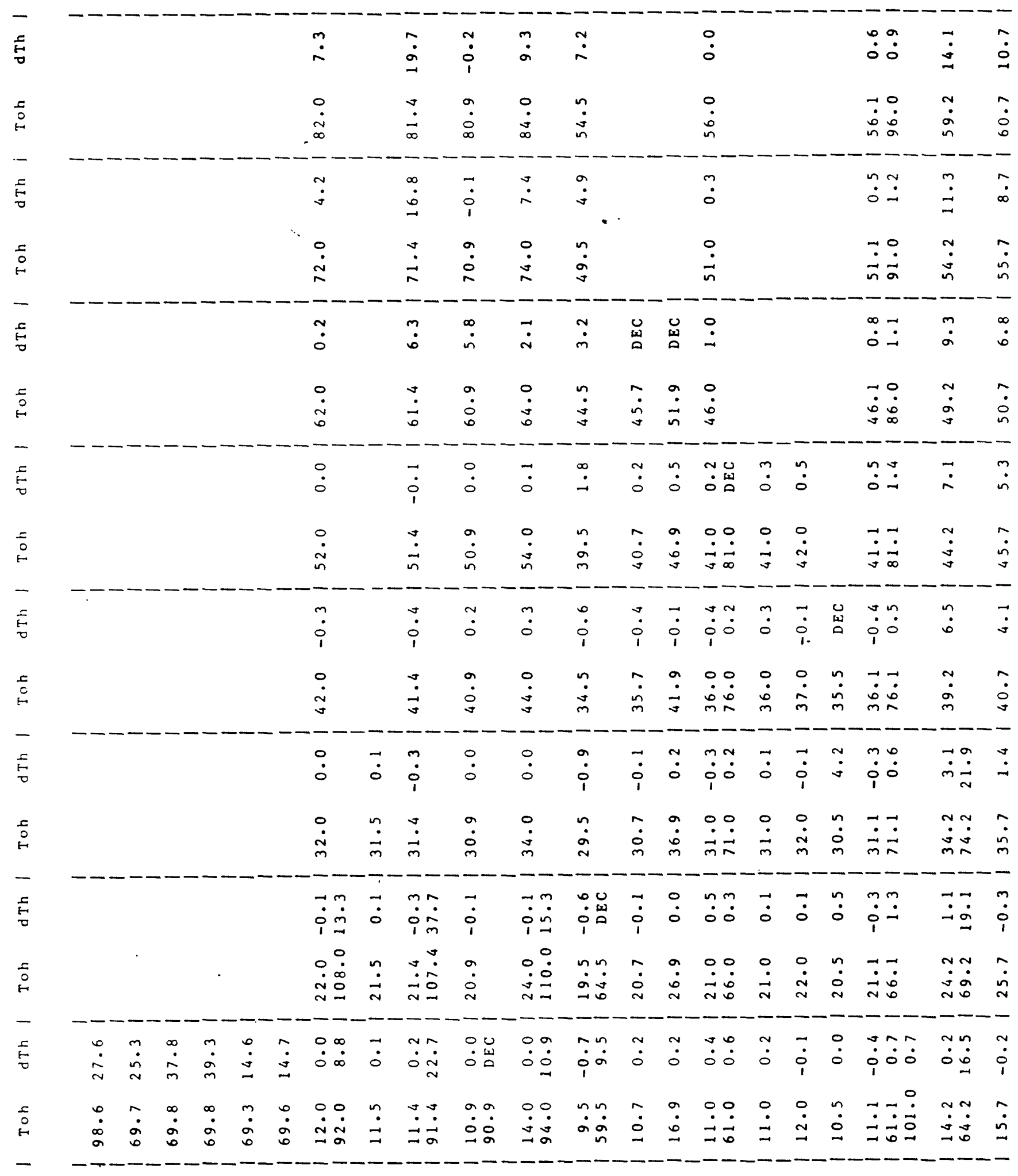

\begin{tabular}{l}
0 \\
0 \\
2 \\
$\vdots$ \\
$z$ \\
$z$ \\
0 \\
0 \\
$w$ \\
3 \\
0 \\
0 \\
$z$ \\
$z$ \\
\hline
\end{tabular}

\begin{tabular}{|c|c|c|c|c|c|c|c|c|c|c|c|c|c|c|c|c|c|c|c|}
\hline$\vec{\infty}$ & $\overrightarrow{0}$ & U & 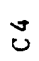 & $\because$ & o & 4 & N & 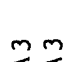 & 2 & $n$ & $\vec{x} \vec{x}$ & $\dddot{x}$ & $\ddot{m}$ & $\underset{\square}{\square}$ & $\ddot{0}$ & $\hat{x}$ & $\stackrel{\infty}{\longleftarrow}$ & $\underset{x}{a} a$ & $\exists=$ \\
\hline $\begin{array}{l}a \\
n \\
n\end{array}$ & in & a & $\begin{array}{l}a \\
n \\
n\end{array}$ & n & $\begin{array}{l}n \\
n \\
1\end{array}$ & 出占 & 岁 & 岇尐 & 㞤嵒 & 出出 & 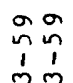 & $\begin{array}{l}a \\
n \\
m\end{array}$ & $\begin{array}{l}a \\
i \\
n\end{array}$ & $\begin{array}{ll}0 & a \\
\text { in } & \text { in } \\
1 & 1\end{array}$ & $\begin{array}{l}a \\
n \\
1 \\
m\end{array}$ & 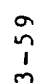 & $\stackrel{a}{n}$ & $\begin{array}{lll}a & a & a \\
\dddot{n} & \sim n & n \\
1 & 1 & 1\end{array}$ & $\begin{array}{ll}a & a \\
n & n \\
1 & 1 \\
m & m\end{array}$ \\
\hline- & 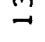 & 2 & $\Rightarrow$ & - & - & $\infty$ & $\sum_{\Sigma}^{\infty}$ & $\sum^{\infty}$ & $\sum_{\Sigma}^{\infty}$ & $\sum \sum$ & -7 & - & - & $\rightarrow-$ & - & - & $\rightarrow$ & $\rightarrow-\rightarrow$ & $\rightarrow-$ \\
\hline$\stackrel{\infty}{\infty}$ & $\underset{\infty}{\infty}$ & $\begin{array}{l}\infty \\
\infty \\
0\end{array}$ & $\begin{array}{l}\infty \\
\infty \\
\infty\end{array}$ & $\underset{\infty}{\infty}$ & $\begin{array}{l}\infty \\
\infty \\
\infty\end{array}$ & $a$ & & $a$ & $\bar{a} \overline{2}$ & $a$ & $\begin{array}{l}\infty \\
\infty \\
\infty \\
\infty \\
0 \\
0 \\
0\end{array}$ & $\begin{array}{l}\infty \\
\infty \\
\infty\end{array}$ & $\begin{array}{l}\infty \\
\infty \\
0\end{array}$ & $\begin{array}{l}\infty \\
\infty \\
\infty \\
0\end{array}$ & $\underset{\infty}{\infty}$ & $\begin{array}{l}\infty \\
\infty \\
\infty\end{array}$ & $\begin{array}{l}\infty \\
\infty \\
0\end{array}$ & $\begin{array}{l}\infty \\
\infty \\
\infty \\
0 \\
0\end{array}$ & $\begin{array}{l}\infty \\
\infty \\
\infty\end{array}$ \\
\hline
\end{tabular}




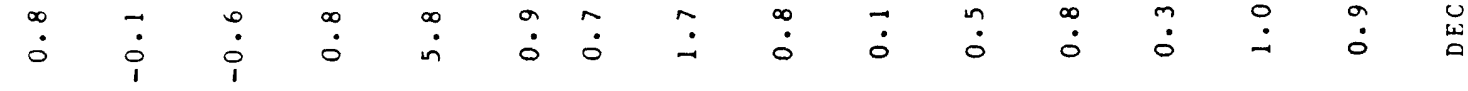

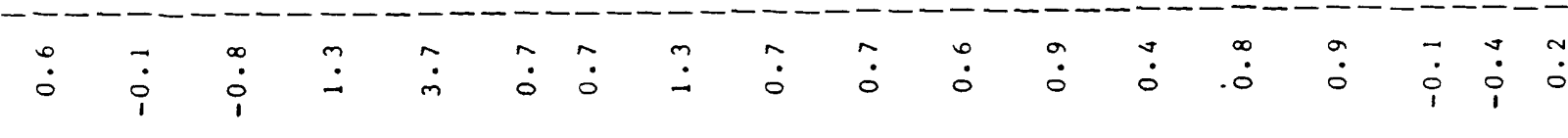

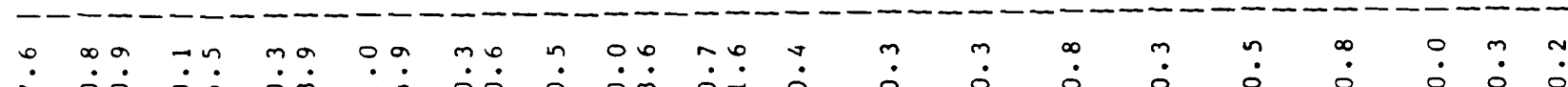

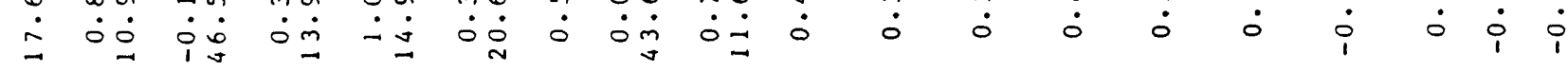

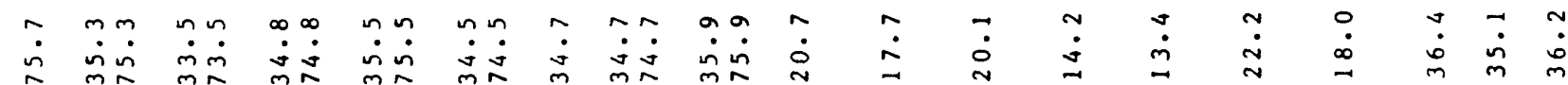
- - - - - - - - - - - - - - - - - - - - - - - - - - - - - -

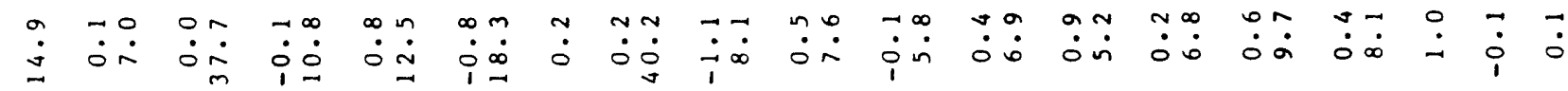

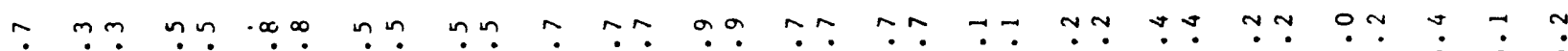

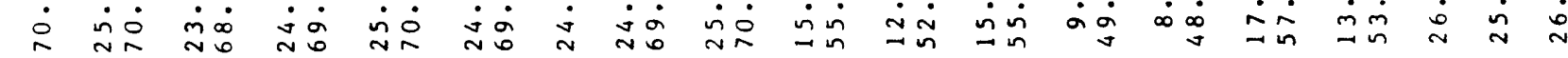
- - - - - - - - - - - - - - - - - - - - - - - - - - - - - - - -

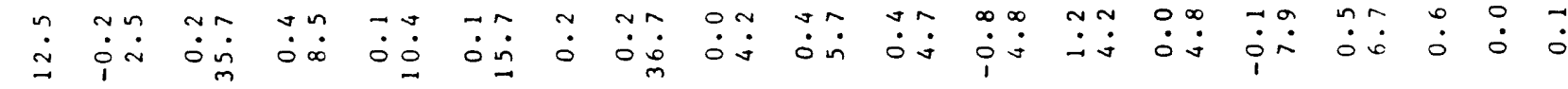

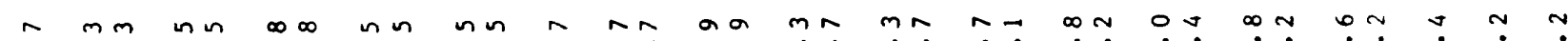

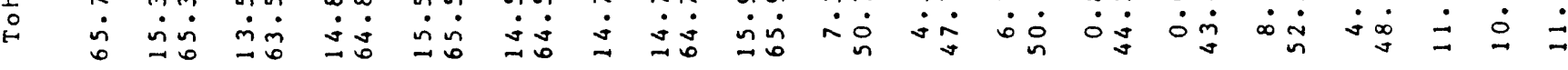

I

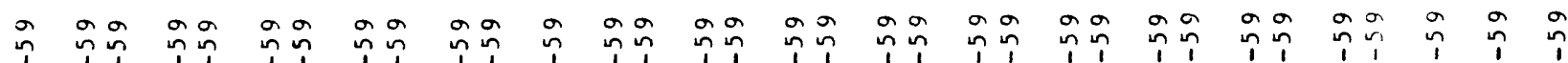

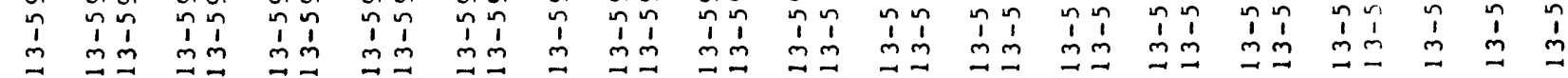

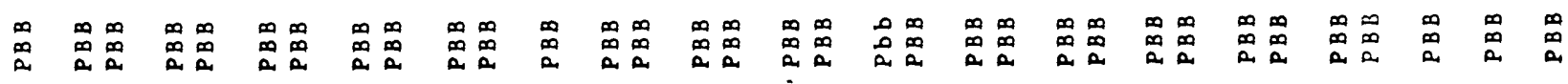




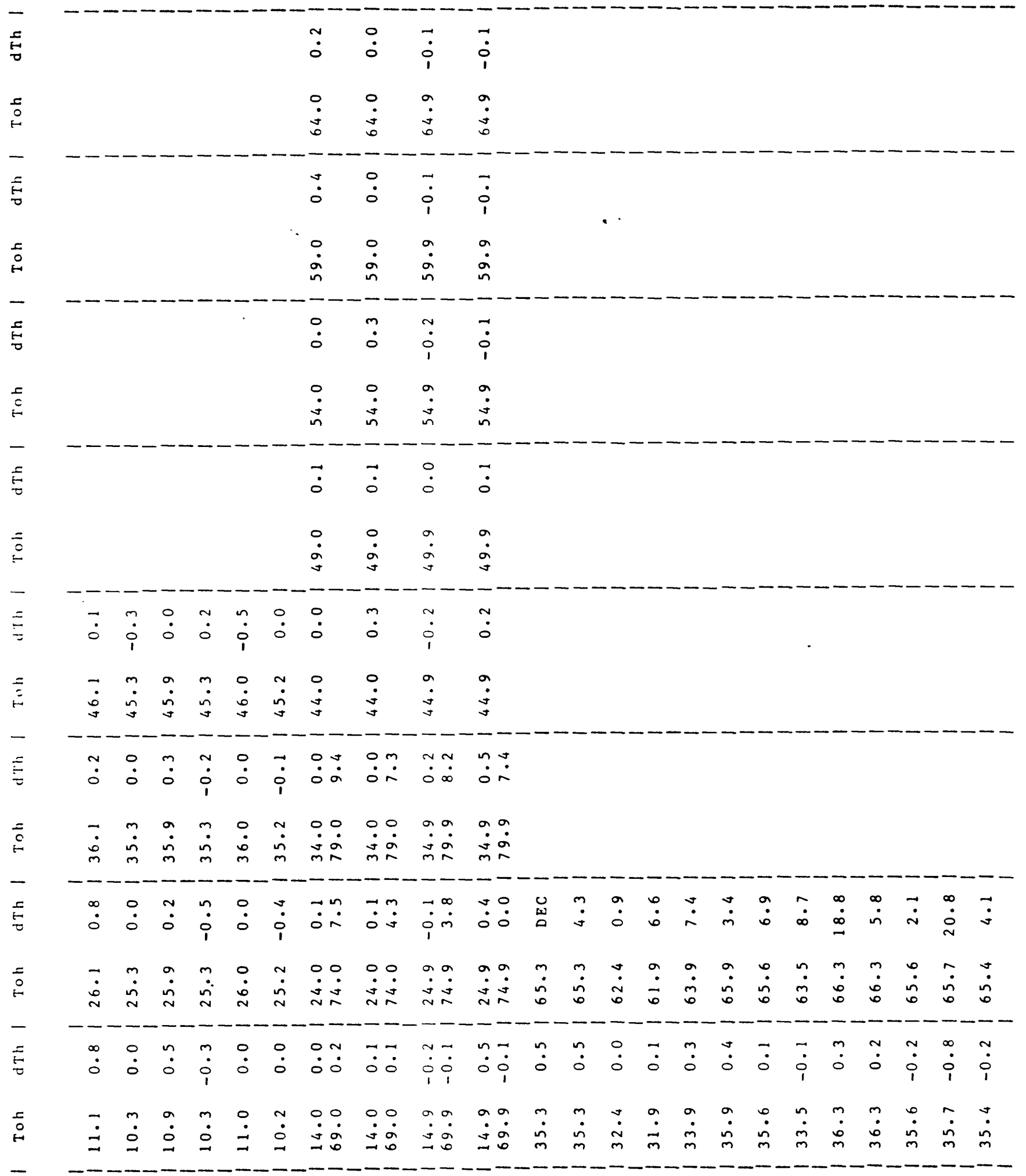

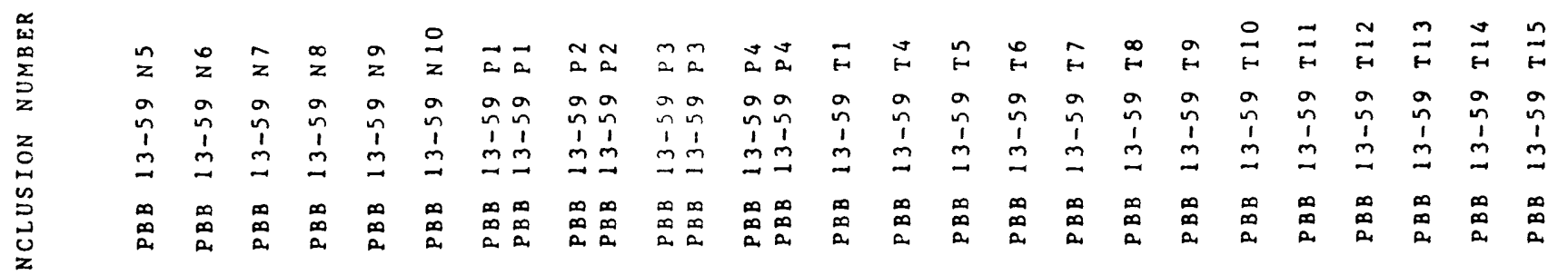




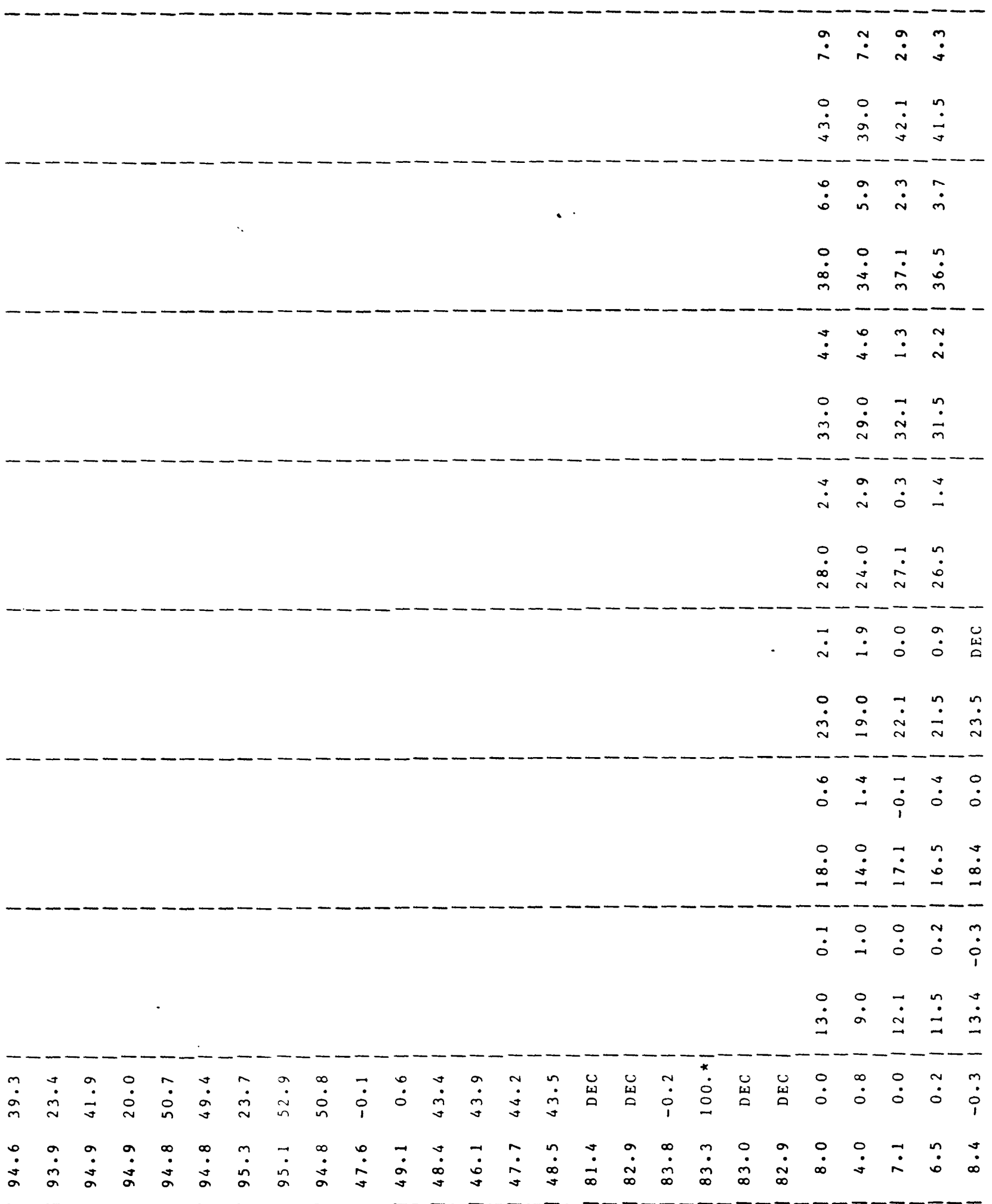

$x$
1
2
3
$z$
$z$
$z$
0
0
0
$\vdots$
0
0
$z$
1

\begin{tabular}{|c|c|c|c|c|c|c|c|c|c|c|c|c|c|c|c|c|c|c|c|c|c|c|c|}
\hline$\tilde{D}$ & $\stackrel{m}{\square}$ & $\vec{D}$ & $\cong$ & $\tilde{s}$ & & $\begin{array}{l}a \\
3\end{array}$ & $\frac{0}{3}$ & $\overline{>}$ & $\stackrel{\sim}{\sim}$ & $\stackrel{m}{>}$ & $\stackrel{n}{>}$ & $\hat{>}$ & $\stackrel{2}{>}$ & 3 & 3 & $\stackrel{\infty}{3}$ & $\frac{0}{3}$ & $\bar{z}$ & $\frac{N}{3}$ & $\vec{x}$ & $\underset{x}{x}$ & $\underset{x}{x}$ & $\vec{x}$ \\
\hline $\begin{array}{l}a \\
\text { nn } \\
1 \\
m\end{array}$ & $\begin{array}{l}a \\
1 \\
m\end{array}$ & $\begin{array}{l}a \\
n \\
1 \\
m\end{array}$ & $\begin{array}{l}9 \\
1 \\
m \\
\end{array}$ & $\begin{array}{l}a \\
\mathfrak{1} \\
m\end{array}$ & $\begin{array}{l}9 \\
\dddot{1} \\
\stackrel{m}{\longrightarrow}\end{array}$ & $\begin{array}{l}a \\
\stackrel{n}{1} \\
m\end{array}$ & $\begin{array}{l}a \\
n \\
m \\
m\end{array}$ & $\begin{array}{l}a \\
1 \\
m\end{array}$ & $\begin{array}{l}a \\
n \\
1 \\
n\end{array}$ & $\begin{array}{l}a \\
n \\
n \\
n\end{array}$ & $\begin{array}{l}a \\
n \\
1 \\
n\end{array}$ & $\begin{array}{l}a \\
\mathfrak{1} \\
n \\
n\end{array}$ & $\stackrel{n}{1}$ & $\begin{array}{l}n \\
n \\
n\end{array}$ & $\begin{array}{l}\text { on } \\
\text { in } \\
\text { m }\end{array}$ & $\begin{array}{l}\text { or } \\
\text { n } \\
1 \\
m \\
0\end{array}$ & $\begin{array}{l}0 \\
\text { ñ } \\
1 \\
n\end{array}$ & $\begin{array}{l}0 \\
n \\
1 \\
n\end{array}$ & $\begin{array}{l}0 \\
\text { in } \\
1 \\
m\end{array}$ & $\begin{array}{l}a \\
n \\
1 \\
n\end{array}$ & $\begin{array}{l}a \\
\text { in } \\
1 \\
m\end{array}$ & $\begin{array}{l}a \\
n \\
n\end{array}$ & $\begin{array}{l}\text { an } \\
1 \\
1 \\
m\end{array}$ \\
\hline $\begin{array}{l}\infty \\
\infty \\
0\end{array}$ & $\begin{array}{l}\infty \\
\infty \\
\infty\end{array}$ & $\begin{array}{l}\infty \\
\text { 品 } \\
0\end{array}$ & $\begin{array}{l}\infty \\
\infty \\
0\end{array}$ & $\infty$ & $\infty$ & $\infty$ & $\begin{array}{l}\infty \\
\infty \\
0\end{array}$ & $\begin{array}{l}\infty \\
\infty \\
\infty\end{array}$ & $\begin{array}{l}\infty \\
\infty \\
\infty\end{array}$ & $\begin{array}{l}\infty \\
\infty \\
0\end{array}$ & $\begin{array}{l}\infty \\
\infty \\
\infty\end{array}$ & $\begin{array}{l}\infty \\
\infty \\
0\end{array}$ & & & $\begin{array}{l}\infty \\
\infty \\
0\end{array}$ & $\begin{array}{l}\infty \\
\infty \\
\infty\end{array}$ & $\begin{array}{l}\infty \\
\infty \\
\infty\end{array}$ & $\begin{array}{l}\infty \\
\infty \\
\infty\end{array}$ & & $\begin{array}{l}\infty \\
\infty \\
\infty\end{array}$ & $\begin{array}{l}\infty \\
\infty \\
0\end{array}$ & $\begin{array}{l}\infty \\
\infty \\
0\end{array}$ & $\begin{array}{l}\infty \\
\infty\end{array}$ \\
\hline
\end{tabular}




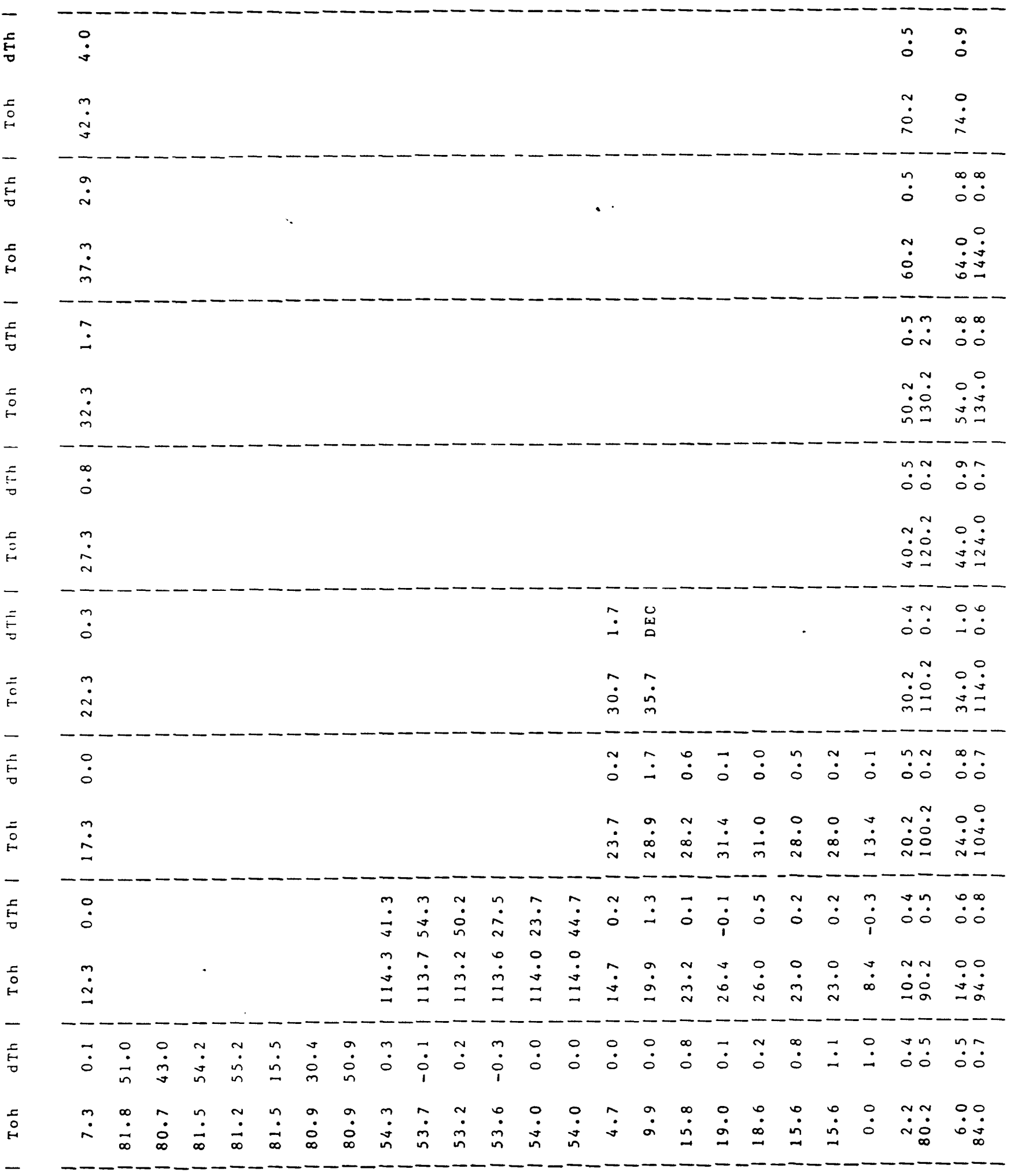

\begin{tabular}{|c|c|c|c|c|c|c|c|c|c|c|c|c|c|c|c|c|c|c|c|c|c|c|}
\hline $\overrightarrow{>}$ & $\underset{\infty}{\sim}$ & $\underset{\nu}{0}$ & aे & $\frac{0}{2}$ & $\stackrel{m}{2}$ & $\stackrel{2}{2}$ & $\vec{N}$ & 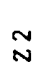 & $\stackrel{N}{N}$ & $\checkmark$ & $\tilde{N}$ & $\stackrel{\sim}{N}$ & $\overrightarrow{<}$ & $\stackrel{\sim}{\tau}$ & $\bar{\infty}$ & $\stackrel{\sim}{\infty}$ & $m$ & $\vec{\infty}$ & $\tilde{n}$ & $\underset{\infty}{\infty}$ & $\vec{u}$ & $\widetilde{U}$ \\
\hline $\begin{array}{l}a \\
n \\
n \\
n\end{array}$ & $\begin{array}{l}a \\
n \\
1 \\
m\end{array}$ & $\begin{array}{l}a \\
n \\
1 \\
m \\
=\end{array}$ & $\begin{array}{l}a \\
n \\
1 \\
m\end{array}$ & $\begin{array}{l}a \\
i \\
m\end{array}$ & $\begin{array}{l}a \\
n \\
m\end{array}$ & $\begin{array}{l}a \\
n \\
1 \\
m\end{array}$ & $\begin{array}{l}a \\
\tilde{1} \\
n\end{array}$ & $\begin{array}{l}a \\
\\
\end{array}$ & $\begin{array}{l}a \\
n \\
n \\
n\end{array}$ & $\begin{array}{l}a \\
n \\
1 \\
m\end{array}$ & $\stackrel{n}{1}$ & $\begin{array}{l}a \\
n \\
1 \\
m\end{array}$ & $\underset{\sim}{\sim}$ & $\underset{n}{\sim}$ & $\underset{\sim}{m}$ & $\underset{\sim}{m}$ & $\begin{array}{l}m \\
\sim \\
\sim \\
\infty\end{array}$ & $\begin{array}{l}n \\
\sim \\
\sim \\
\infty\end{array}$ & $\underset{m}{m}$ & $\begin{array}{l}m \\
\sim \\
\sim \\
\infty\end{array}$ & $\begin{array}{l}\underset{m}{m} \underset{n}{m} \\
\underset{n}{n} \tilde{m} \\
m\end{array}$ & 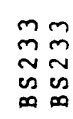 \\
\hline $\begin{array}{l}\infty \\
\infty \\
0\end{array}$ & $\begin{array}{l}\infty \\
\infty \\
0\end{array}$ & $\begin{array}{l}\infty \\
\infty \\
\infty\end{array}$ & $\begin{array}{l}\infty \\
\infty \\
0\end{array}$ & $\begin{array}{l}\infty \\
\infty \\
\infty\end{array}$ & $\begin{array}{l}\infty \\
\infty \\
0\end{array}$ & $\begin{array}{l}\infty \\
\infty \\
\infty\end{array}$ & $\stackrel{\infty}{\infty}$ & $\begin{array}{l}\infty \\
\infty \\
\infty\end{array}$ & $\begin{array}{l}\infty \\
\infty \\
\infty\end{array}$ & $\infty$ & $\infty$ & $\begin{array}{l}\infty \\
\infty \\
\infty\end{array}$ & $\sum_{\alpha}^{\infty}$ & $\sum_{\alpha}^{\infty}$ & $\sum_{\alpha}^{\infty}$ & $\sum_{\infty}^{\infty}$ & $\sum_{\infty}^{\infty}$ & $\sum_{\infty}^{\infty}$ & $\sum_{R}^{\infty}$ & $\sum_{\Omega}^{\infty}$ & $\sum_{a}^{\infty} \sum_{a}^{\infty}$ & $\sum_{a}^{\infty} \sum_{n}^{\infty}$ \\
\hline
\end{tabular}




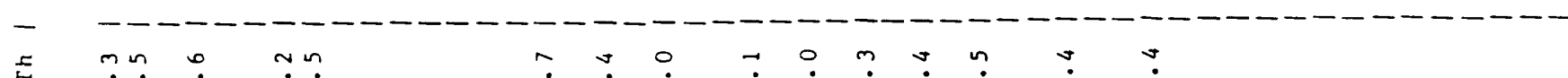

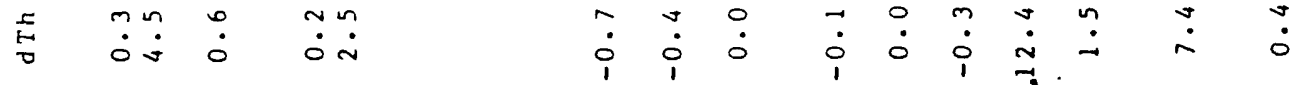

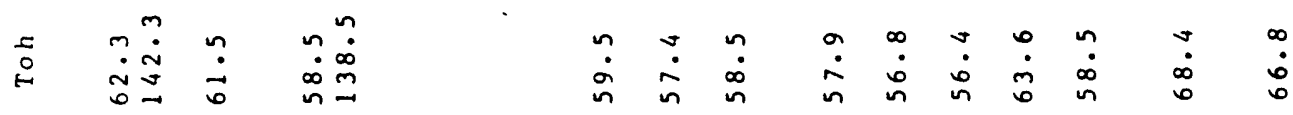

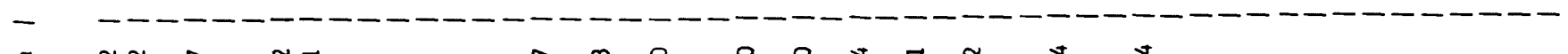

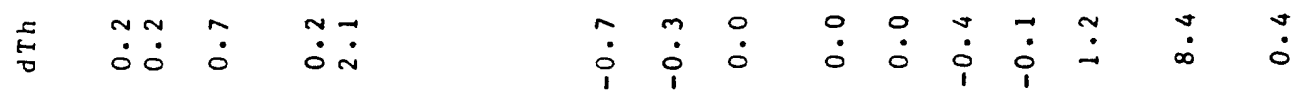

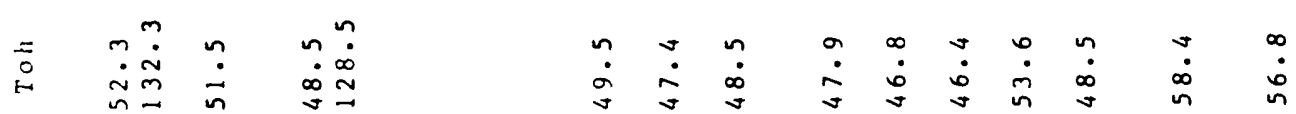

- mm no no

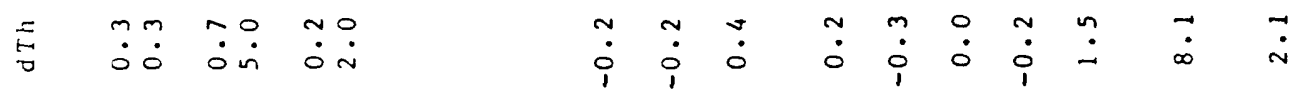

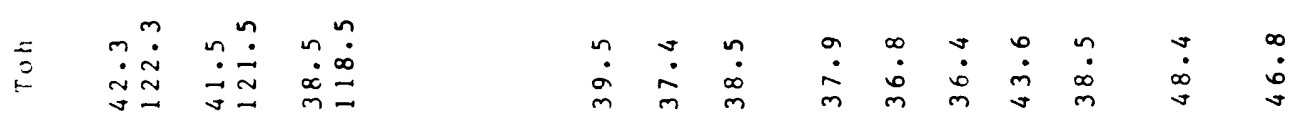

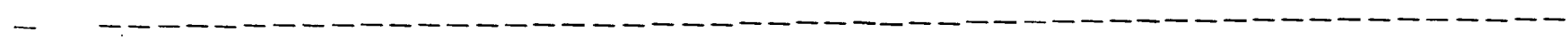

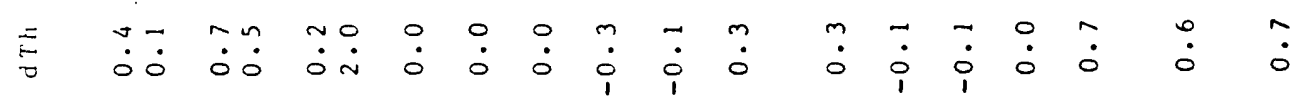

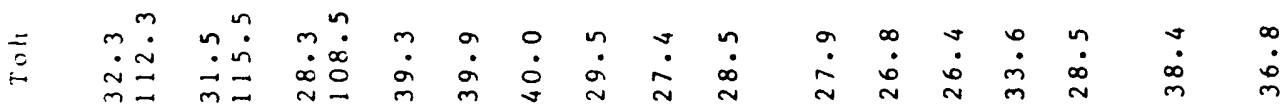

- - - - - - - -

$=$ Jm n

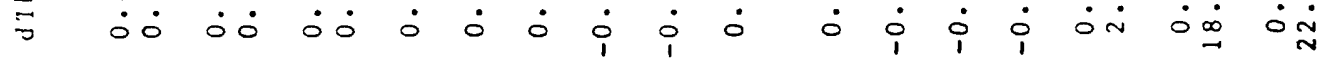

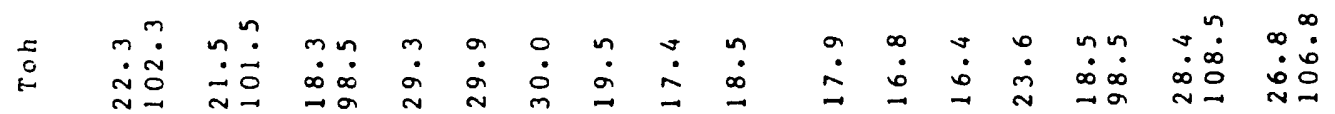

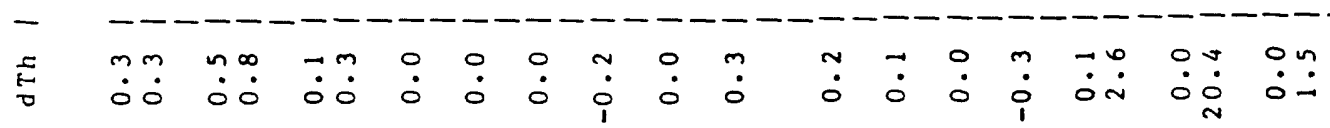

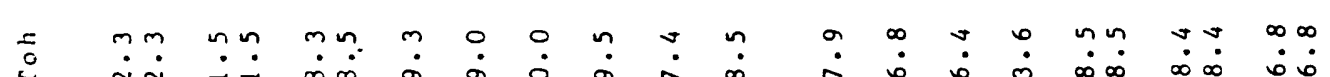

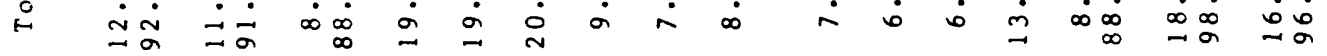

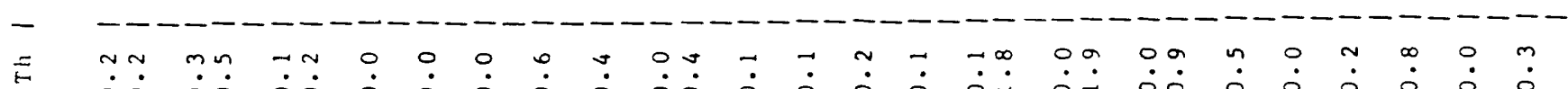

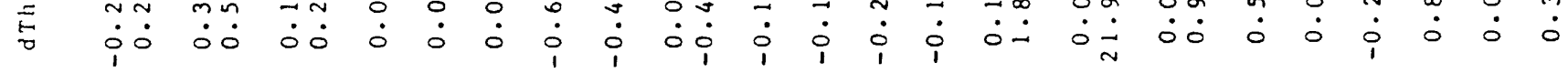

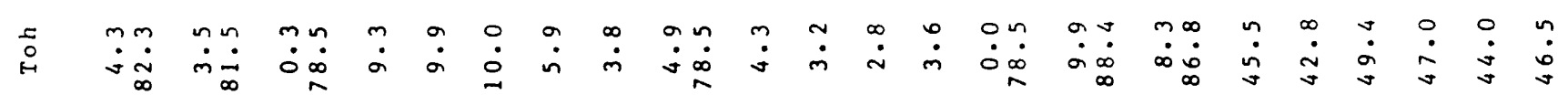

- - - - - - - - - - - - - - - - - - - - - - - - - - - - - - - - - - - - - - - - - - - - - - - - -

$x$
0
2
$\Sigma$
$z$
$z$
$z$
0
0
0
$\vdots$
0
$z$
$=$

\begin{tabular}{|c|c|c|c|c|c|c|c|c|c|c|c|c|c|c|c|c|c|c|c|c|c|}
\hline$m m$ & $\checkmark v$ & n & $\overrightarrow{0}$ & $N$ & $m$ & & & & & & & & & & & & & & & & \\
\hline & & & & $a$ & $a$ & - & $N$ & $m m$ & $\checkmark$ & n & 0 & $n$ & $\rightarrow-$ & $\sim N$ & $m m$ & $\overrightarrow{.}$ & N & $m$ & $\checkmark$ & $n$ & 0 \\
\hline$m m$ & $m m$ & $m m$ & $m$ & $m$ & $m$ & $\varangle$ & $<$ & $<<$ & $\ll$ & $<$ & $\varangle$ & $<$ & $\infty \infty$ & $\approx \infty$ & $\infty \infty$ & 0 & 0 & 0 & 0 & 0 & 0 \\
\hline$\underset{\sim}{N} \sim$ & $\begin{array}{l}\sim \\
\sim \\
\infty\end{array}$ & $\begin{array}{ll}N & N \\
\infty & \infty\end{array}$ & $\underset{\sim}{\sim}$ & $\underset{\infty}{\infty}$ & $\underset{\infty}{\sim}$ & $\begin{array}{l}x \\
a \\
z\end{array}$ & $\begin{array}{l}x \\
z\end{array}$ & $\begin{array}{ll}x & x \\
a & a \\
z & z\end{array}$ & $\begin{array}{l}x \\
a \\
z\end{array}$ & $\begin{array}{l}x \\
a \\
z \\
z\end{array}$ & $\begin{array}{l}x \\
z \\
z\end{array}$ & $\begin{array}{l}x \\
a \\
z \\
z\end{array}$ & $\begin{array}{l}x \not x \\
a n \\
z z \\
z z\end{array}$ & $\begin{array}{l}x x \\
a \\
a \\
z z \\
z\end{array}$ & $\begin{array}{l}x x \\
D a \\
z z\end{array}$ & $\begin{array}{l}x \\
a \\
z\end{array}$ & $\begin{array}{l}x \\
2 \\
z\end{array}$ & $\begin{array}{l}x \\
0 \\
2 \\
z\end{array}$ & $\begin{array}{l}x \\
a \\
z \\
z\end{array}$ & $\begin{array}{l}x \\
a \\
z \\
z\end{array}$ & $\begin{array}{l}x \\
0 \\
z \\
z\end{array}$ \\
\hline$\sum_{n}^{\infty} \sum_{n}^{\infty}$ & $\sum_{a}^{\infty} \sum_{n}^{\infty}$ & $\sum_{n}^{\infty} \sum_{n}^{\infty}$ & $\sum_{0}^{\infty}$ & $\sum_{n}^{\infty}$ & $\sum_{0}^{\infty}$ & & & & & & & & & & & & & & & & \\
\hline
\end{tabular}



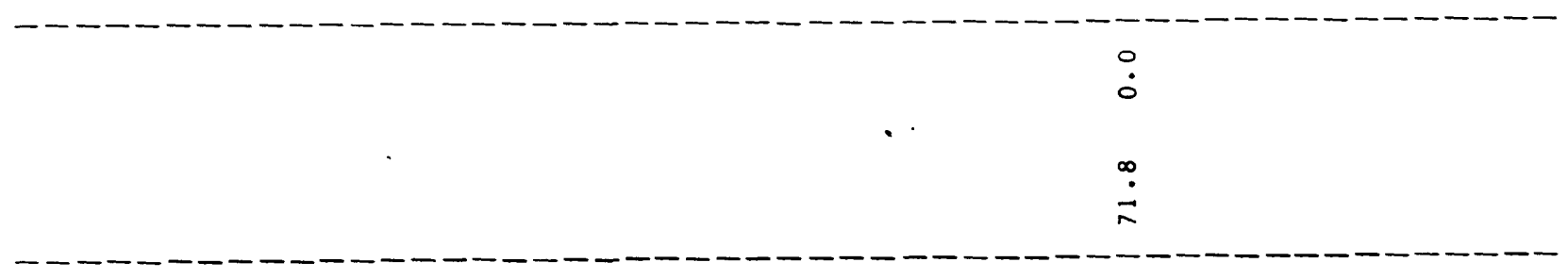

$\overrightarrow{0} \quad \dot{0}$

$\stackrel{1}{\circ}$

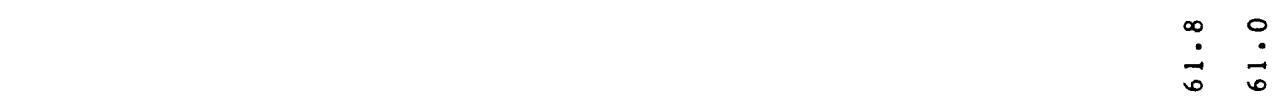

$=$

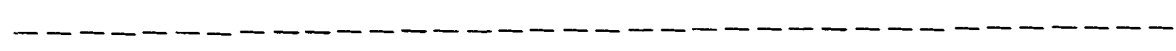

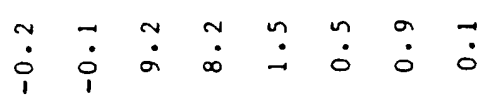

$=$

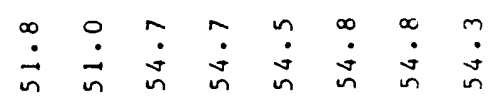

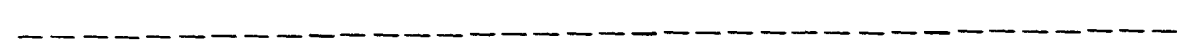

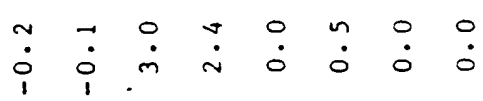

$\frac{1}{3}$

$\frac{1}{2}$

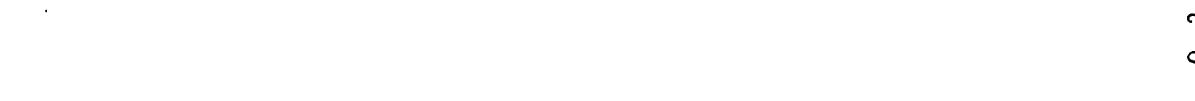

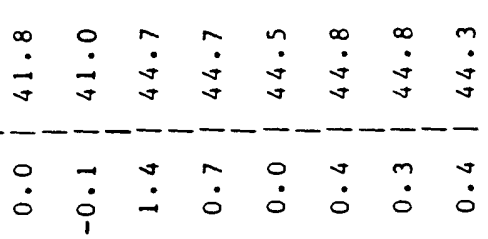

옹

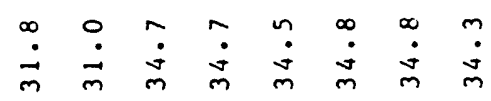

E

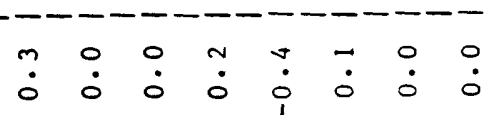

둥

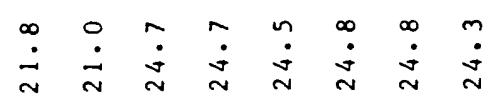

-

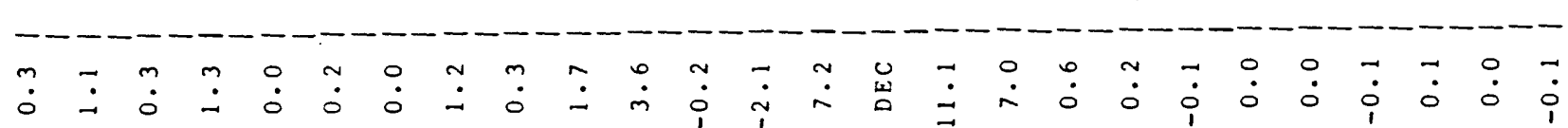




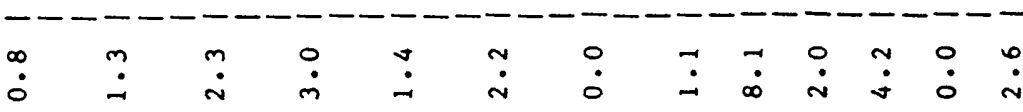

$\stackrel{2}{\circ}$

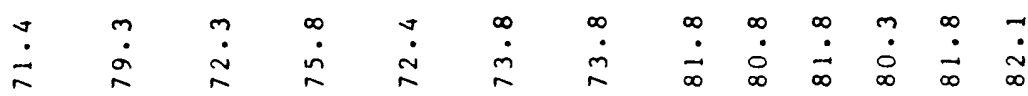

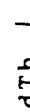

$\underset{0}{2}$

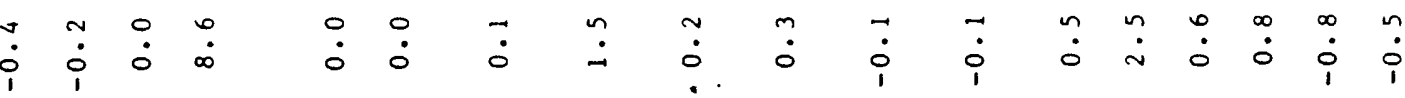

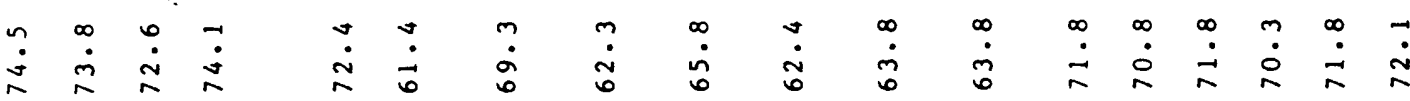
$\underset{D}{-}$

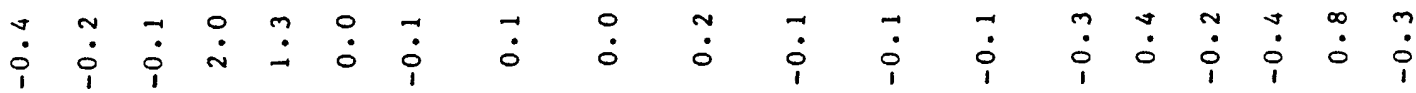

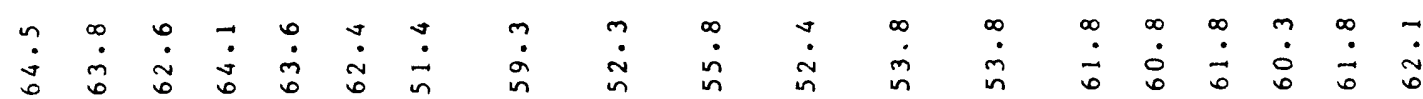

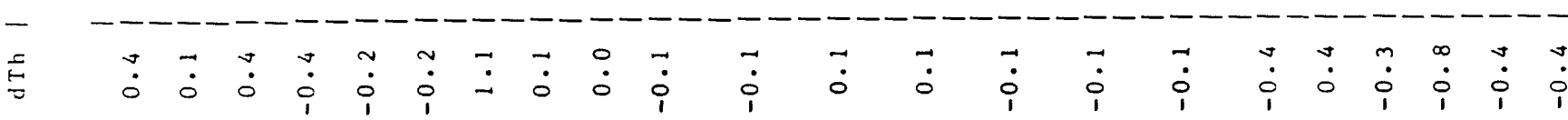

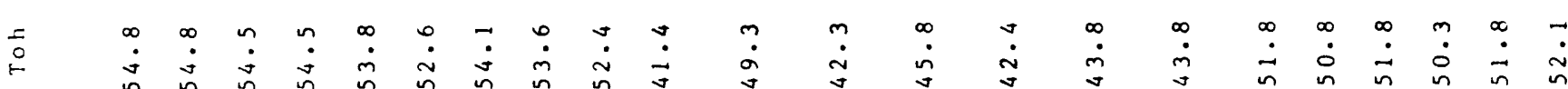
- - - - - - - - - - - - - - - - - - - - - - - - - - - - - - - - - - - - - $=$

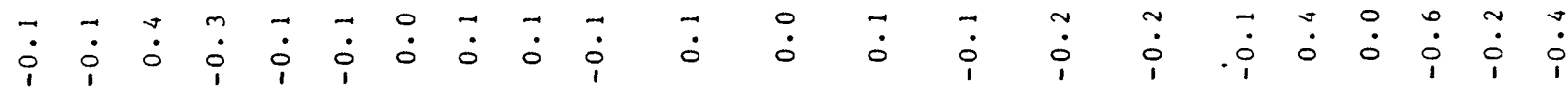

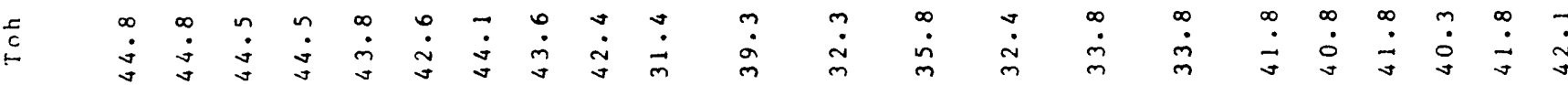

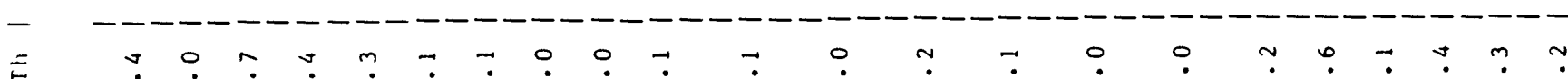

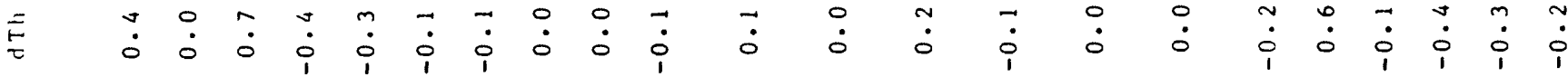

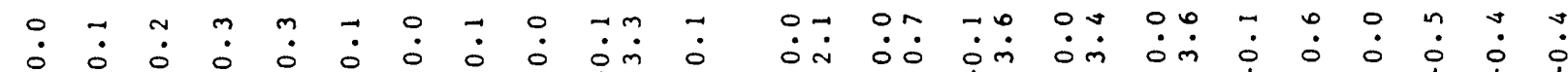

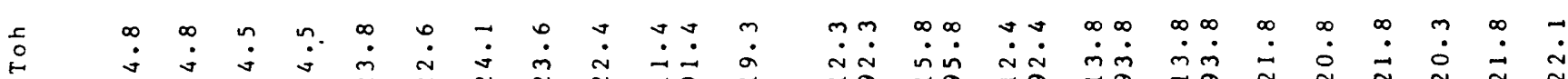

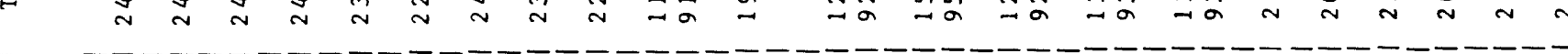




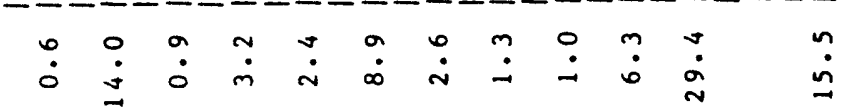

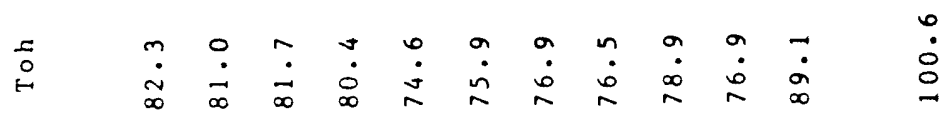

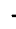

to

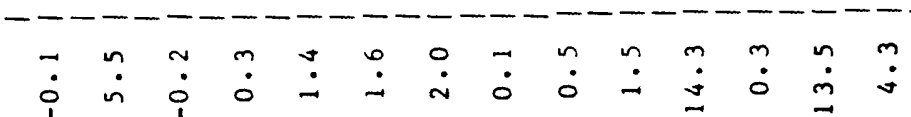

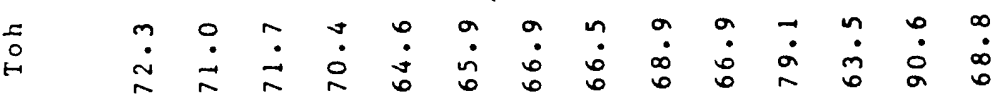

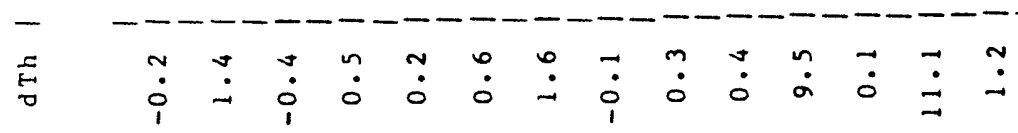

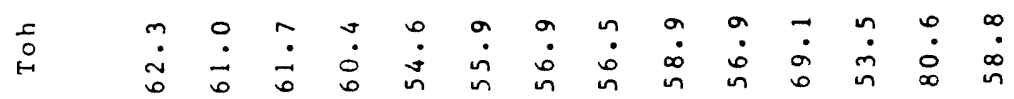

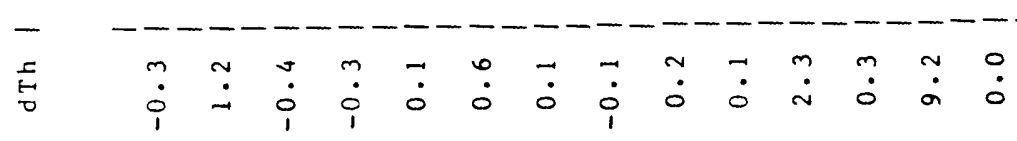

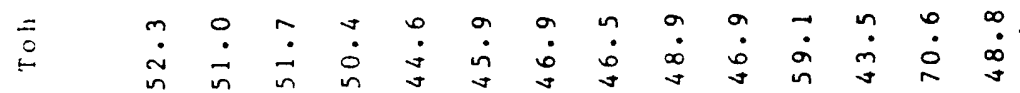

- - - - - - - - - - - - - - - - - - - - - -

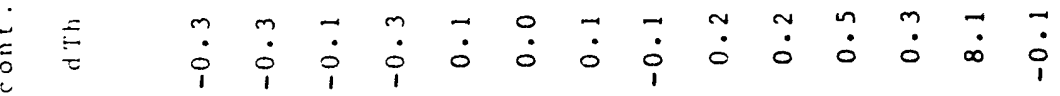

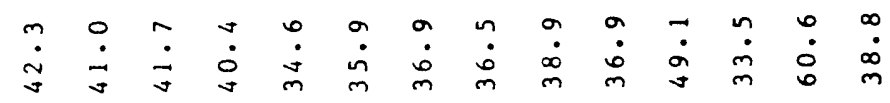

- - - - - - - - - - - - - - - - - - - -

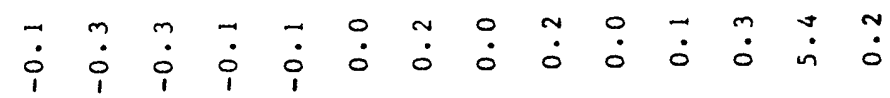




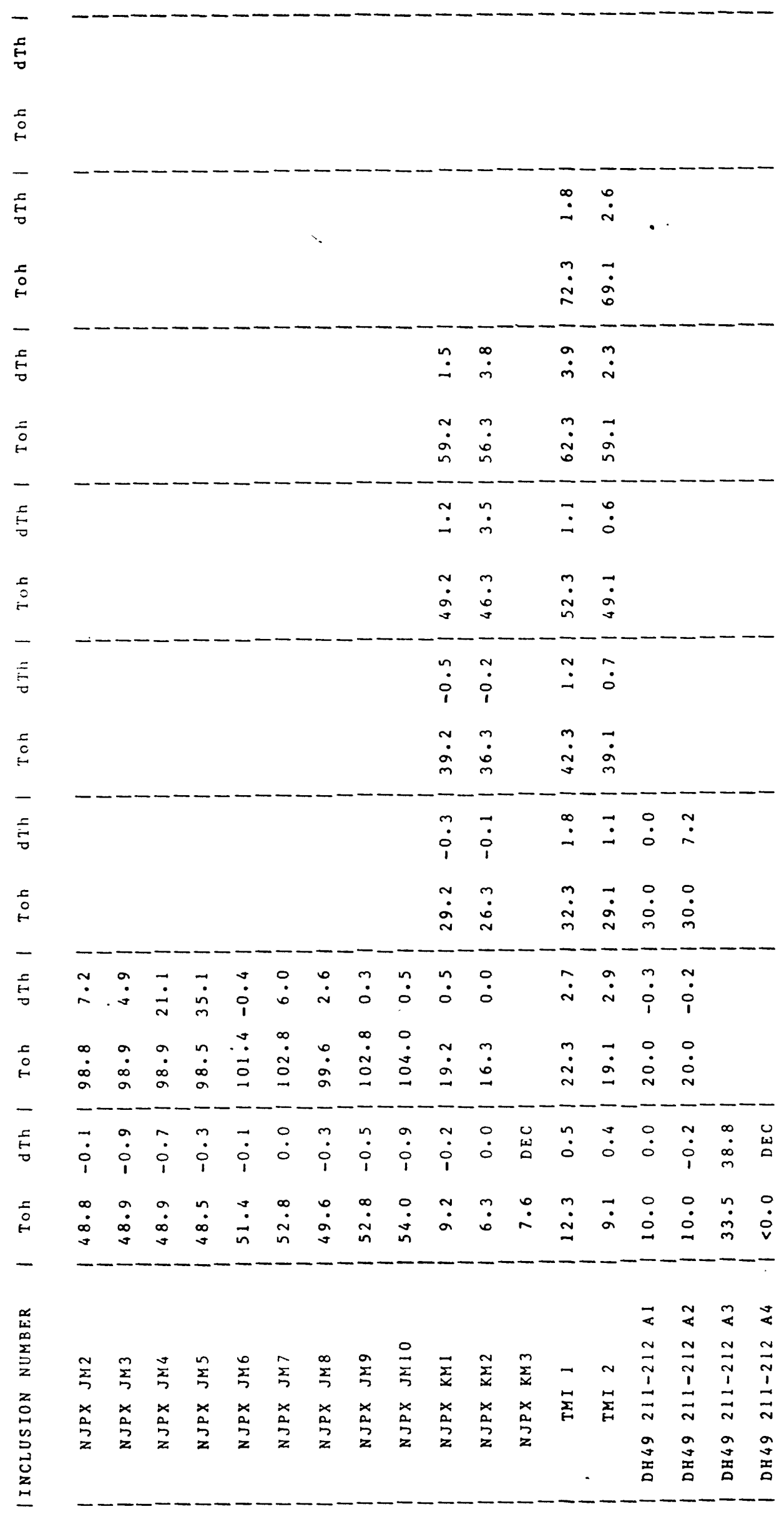

